\title{
Sum rules for the supersymmetric eight-vertex model
}

\author{
SANDRINE BRASsEur and Christian HAgEndorF \\ Université catholique de Louvain \\ Institut de Recherche en Mathématique et Physique \\ Chemin du Cyclotron 2, 1348 Louvain-la-Neuve, Belgium \\ sandrine.brasseur@uclouvain.be, christian.hagendorf@uclouvain.be
}

\begin{abstract}
The eight-vertex model on the square lattice with vertex weights $a, b, c, d$ obeying the relation $\left(a^{2}+a b\right)\left(b^{2}+a b\right)=\left(c^{2}+a b\right)\left(d^{2}+a b\right)$ is considered. Its transfer matrix with $L=2 n+1, n \geqslant 0$, vertical lines and periodic boundary conditions along the horizontal direction has the doubly-degenerate eigenvalue $\Theta_{n}=(a+b)^{2 n+1}$. A basis of the corresponding eigenspace is investigated. Several scalar products involving the basis vectors are computed in terms of a family of polynomials introduced by Rosengren and Zinn-Justin. These scalar products are used to find explicit expressions for particular entries of the vectors. The proofs of these results are based on the generalisation of the eigenvalue problem for $\Theta_{n}$ to the inhomogeneous eight-vertex model.
\end{abstract}

\section{Introduction}

In the present work, we pursue an investigation of the square lattice eight-vertex model whose vertex weights $a, b, c, d$ obey the relation

$$
\left(a^{2}+a b\right)\left(b^{2}+a b\right)=\left(c^{2}+a b\right)\left(d^{2}+a b\right) .
$$

We consider the model on a cylinder with $L \geqslant 1$ vertical lines and periodic boundary conditions along the horizontal direction. If the number of vertical lines $L=2 n+1, n \geqslant 0$, is odd, then the transfer matrix of the vertex model possesses the remarkably simple doubly-degenerate eigenvalue

$$
\Theta_{n}=(a+b)^{2 n+1} .
$$

The existence of this eigenvalue was first conjectured by Stroganov [1]. Recently, Liénardy and Hagendorf proved this conjecture with the help of supersymmetry techniques $[2,3]$. Because of the supersymmetry, we refer to the case where the vertex weights obey (1.1) as the supersymmetric eight-vertex model. Several investigations, inspired by the simple form of $\Theta_{n}$, have revealed exciting connections between the supersymmetric eight-vertex model and a variety of topics in mathematical physics. Examples include elliptic functional equations [4-6], the Painlevé VI equation $[7,8]$, and special polynomials [9-12].

Our main objective is to investigate the eigenspace of $\Theta_{n}$. Bazhanov and Mangazeev [13], and Razumov and Stroganov [14] initiated this investigation by exploiting the fact that the eigenvectors of $\Theta_{n}$ are the ground-state vectors of the Hamiltonian of a XYZ quantum spin chain. They computed these vectors for small $n$ with the help of exact diagonalisation methods applied to the spin-chain Hamiltonian. This computation led them to numerous conjectures on the eigenvectors, providing explicit expressions for their components and for scalar products. The 
latter are often referred to as sum rules. Subsequently, a rigorous investigation of the eigenspace for general $n$ was undertaken by Zinn-Justin [15]. He considered a generalisation of the eigenvalue problem for $\Theta_{n}$ to the inhomogeneous eight-vertex model. Based on only one conjecture about its solution, he was able to derive one of the sum rules that had been observed by Bazhanov and Mangazeev. In this article, we use Zinn-Justin's conjecture to investigate the eigenspace of $\Theta_{n}$ in further detail. To this end, we apply a well-established induction technique of Izergin and Korepin's $[16,17]$. It allows us to derive explicit expressions for several scalar products of an eigenvector of the transfer matrix of the inhomogeneous eight-vertex model. From their homogeneous limits, we deduce several new sum rules and exact expressions for the components of the eigenvectors of $\Theta_{n}$.

The layout of this article is the following. In Section 2, we recall the definition of the transfer matrix of the inhomogeneous eight-vertex model. Focussing on the supersymmetric case, we discuss a generalisation of the eigenvalue problem for $\Theta_{n}$ to the inhomogeneous model and the properties of its solution that follow from Zinn-Justin's conjecture. In Section 3, we introduce scalar products involving this solution as well as several solutions to the boundary Yang-Baxter equation. We obtain exact expressions for these scalar products in terms of the so-called elliptic Tsuchiya determinant. In Section 4, we compute the homogeneous limit of these expressions. We use them to derive and analyse several properties of the eigenvectors of $\Theta_{n}$. We present our conclusions in Section 5.

\section{The inhomogeneous eight-vertex model}

In this section, we review known results on the eight-vertex model. We define the notations and conventions that we use throughout this article in Section 2.1. In Section 2.2, we recall the definition of the transfer matrix of the inhomogeneous eight-vertex model, and some of its properties. From Section 2.3 on, we focus on the supersymmetric eight-vertex model. We recall a generalisation of the eigenvalue problem for $\Theta_{n}$ as well as Zinn-Justin's conjecture on its solution. Moreover, we review several known properties of this solution.

\section{$2.1 \quad$ Notations}

Throughout this article, $L$ denotes a positive integer. Let us consider the space $V^{L}=V_{1} \otimes \cdots \otimes V_{L}$, where $V_{i}=\mathbb{C}^{2}$ for each $i=1, \ldots, L$. Its canonical basis vectors are labelled by sequences $\boldsymbol{\alpha}=\alpha_{1} \cdots \alpha_{L}$, with $\alpha_{i} \in\{\uparrow, \downarrow\}$ for each $i=1, \ldots, L$. We refer to these sequences as spin configurations. The basis vector $|\boldsymbol{\alpha}\rangle$ corresponding to the spin configuration $\boldsymbol{\alpha}$ is

$$
|\boldsymbol{\alpha}\rangle=\left|\alpha_{1}\right\rangle \otimes \cdots \otimes\left|\alpha_{L}\right\rangle,
$$

where

$$
|\uparrow\rangle=\left(\begin{array}{l}
1 \\
0
\end{array}\right), \quad|\downarrow\rangle=\left(\begin{array}{l}
0 \\
1
\end{array}\right) .
$$

Every vector $|\psi\rangle \in V^{L}$ can be expanded along the basis vectors:

$$
|\psi\rangle=\sum_{\boldsymbol{\alpha}} \psi_{\boldsymbol{\alpha}}|\boldsymbol{\alpha}\rangle
$$

We refer to the numbers $\psi_{\boldsymbol{\alpha}}$ in this expansion as the components of $|\psi\rangle$. Furthermore, for every vector $|\phi\rangle \in V^{L}$, we define a dual vector by transposition $\langle\phi|=| \phi\rangle^{t}$. The corresponding dual pairing is

$$
\langle\phi \mid \psi\rangle=\sum_{\boldsymbol{\alpha}} \phi_{\boldsymbol{\alpha}} \psi_{\boldsymbol{\alpha}}
$$


It defines a (real) scalar product on the subspace of $V^{L}$ of vectors with real components. We denote by $\|\psi\|^{2}=\langle\psi \mid \psi\rangle$ the square norm of a vector in this subspace. For simplicity, we use the term 'scalar product' and 'square norm' even for vectors outside this subspace.

Let $M$ be an integer with $1 \leqslant M \leqslant L$, and consider a linear operator $A \in$ End $V^{M}$. For each choice of integers $1 \leqslant i_{1}<\cdots<i_{M} \leqslant L$, we denote by $A_{i_{1}, \ldots, i_{M}}$ the canonical embedding of $A$ into End $V^{L}$. It acts nontrivially only on the factors $V_{i_{1}}, \ldots, V_{i_{M}}$ of $V^{L}$, and acts like the identity operator on all other factors. As an example, we consider the case where $M=1$ and $A$ is one of the Pauli matrices

$$
\sigma^{x}=\left(\begin{array}{ll}
0 & 1 \\
1 & 0
\end{array}\right), \quad \sigma^{y}=\left(\begin{array}{cc}
0 & -\mathrm{i} \\
\mathrm{i} & 0
\end{array}\right), \quad \sigma^{z}=\left(\begin{array}{cc}
1 & 0 \\
0 & -1
\end{array}\right)
$$

On $V^{L}$, we have

$$
\sigma_{i}^{\kappa}=\underbrace{\mathbb{1} \otimes \cdots \otimes \mathbb{1}}_{i-1} \otimes \sigma^{\kappa} \otimes \underbrace{\mathbb{1} \otimes \cdots \otimes \mathbb{1}}_{L-i}, \quad \kappa=x, y, z,
$$

for each $i=1, \ldots, L$, where $\mathbb{1}$ denotes the $2 \times 2$ identity matrix. In terms of these operators, we define the spin-parity operator $P$ and the spin-reversal operator $F$ as

$$
P=(-1)^{L} \prod_{i=1}^{L} \sigma_{i}^{z}, \quad F=\prod_{i=1}^{L} \sigma_{i}^{x}
$$

They obey the relation

$$
F P=(-1)^{L} P F
$$

\subsection{The transfer matrix}

\section{The $R$-matrix}

The eight-vertex model is an integrable vertex model of planar statistical mechanics that can be constructed from an $R$-matrix [18]. This $R$-matrix is an operator $R \in$ End $V^{2}$. In the basis $\{|\uparrow \uparrow\rangle,|\uparrow \downarrow\rangle,|\downarrow \uparrow\rangle,|\downarrow \downarrow\rangle\}$ it acts like the matrix

$$
R=\left(\begin{array}{llll}
a & 0 & 0 & d \\
0 & b & c & 0 \\
0 & c & b & 0 \\
d & 0 & 0 & a
\end{array}\right)
$$

Here, the numbers $a, b, c, d$ are the vertex weights. It is often convenient to use a parameterisation of these weights in terms of the Jacobi theta functions $\vartheta_{i}(u)=\vartheta_{i}(u, p), i=1, \ldots, 4 .^{1}$ In our parameterisation, the weights depend on a spectral parameter $u$, the crossing parameter $\eta$ and the elliptic nome $p=\mathrm{e}^{\mathrm{i} \pi \tau}, \operatorname{Im} \tau>0$. Up to an irrelevant overall factor, they are given by

$$
\begin{aligned}
a(u) & =\vartheta_{4}\left(2 \eta, p^{2}\right) \vartheta_{1}\left(u+2 \eta, p^{2}\right) \vartheta_{4}\left(u, p^{2}\right), \\
b(u) & =\vartheta_{4}\left(2 \eta, p^{2}\right) \vartheta_{4}\left(u+2 \eta, p^{2}\right) \vartheta_{1}\left(u, p^{2}\right), \\
c(u) & =\vartheta_{1}\left(2 \eta, p^{2}\right) \vartheta_{4}\left(u+2 \eta, p^{2}\right) \vartheta_{4}\left(u, p^{2}\right), \\
d(u) & =\vartheta_{1}\left(2 \eta, p^{2}\right) \vartheta_{1}\left(u+2 \eta, p^{2}\right) \vartheta_{1}\left(u, p^{2}\right) .
\end{aligned}
$$

\footnotetext{
${ }^{1}$ We use the classical notation of Whittaker and Watson for the Jacobi theta functions. Throughout this article, we often omit the intermediate steps of our calculations that involve the many identities between these functions $[19,20]$.
} 
Here and in the following, we only write out the dependence on the spectral parameter $u$, but not on $\eta$ and $p$. The parameterisation implies that the $R$-matrix obeys the so-called Yang-Baxter equation. On $V^{3}$, it is given by

$$
R_{1,2}(u-v) R_{1,3}(u) R_{2,3}(v)=R_{2,3}(v) R_{1,3}(u) R_{1,2}(u-v) .
$$

Moreover, we note that $R(0)=\vartheta_{4}\left(0, p^{2}\right) \vartheta_{1}\left(2 \eta, p^{2}\right) \vartheta_{4}\left(2 \eta, p^{2}\right) \mathcal{P}$, where $\mathcal{P}$ is the permutation operator on $V^{2}$, acting on the basis vectors as $\mathcal{P}\left|\alpha_{1} \alpha_{2}\right\rangle=\left|\alpha_{2} \alpha_{1}\right\rangle$. We use it to define the $\check{R}$-matrix $\check{R}(u)=\mathcal{P} R(u)$. It obeys the braid form of the Yang-Baxter equation

$$
\check{R}_{1,2}(u-v) \check{R}_{2,3}(u) \check{R}_{1,2}(v)=\check{R}_{2,3}(v) \check{R}_{1,2}(u) \check{R}_{2,3}(u-v)
$$

\section{The transfer matrix}

We now consider a square lattice with $L$ vertical lines and periodic boundary conditions along the horizontal direction. The transfer matrix of the eight-vertex model on this lattice is

$$
T\left(u \mid u_{1}, \ldots, u_{L}\right)=\operatorname{tr}_{0}\left(R_{0, L}\left(u_{L}-u\right) \cdots R_{0,2}\left(u_{2}-u\right) R_{0,1}\left(u_{1}-u\right)\right) .
$$

In this expression, the $R$-matrices act on the tensor product $V_{0} \otimes V^{L}$, where $V_{0}=\mathbb{C}^{2}$ is called the auxiliary space. The trace is taken over this auxiliary space. We refer to $u$ as the spectral parameter and $u_{1}, \ldots, u_{L}$ as the inhomogeneity parameters. If $u_{1}=\cdots=u_{L}=0$, then we call the eight-vertex model described by this transfer matrix homogeneous, otherwise inhomogeneous.

The transfer matrix is diagonalisable, at least for real $u, \eta, p$ and real $u_{1}, \ldots, u_{L}$ sufficiently close to 0 [18]. It is possible to choose its eigenvectors to be independent of the spectral parameter $u$. This property follows from the commutation relation

$$
\left[T\left(u \mid u_{1}, \ldots, u_{L}\right), T\left(v \mid u_{1}, \ldots, u_{L}\right)\right]=0,
$$

for all $u, v$, which itself is a consequence of the Yang-Baxter equation (2.11) [18]. Moreover, the transfer matrix possesses two elementary symmetries: It is invariant under spin reversal and preserves the spin parity. These symmetries lead to the commutation relations

$$
\left[T\left(u \mid u_{1}, \ldots, u_{L}\right), F\right]=\left[T\left(u \mid u_{1}, \ldots, u_{L}\right), P\right]=0 .
$$

We note that for odd $L$, the relations (2.8) and (2.15) imply that each transfer-matrix eigenvalue has an even degeneracy.

\subsection{The supersymmetric point}

Unless stated otherwise, we consider throughout the remainder of this article the case where $L=2 n+1$, with $n \geqslant 0$ an integer, and

$$
\eta=\pi / 3 .
$$

For this value of the crossing parameter, the weights (2.10) obey the defining relation (1.1) of the supersymmetric eight-vertex model. Moreover, the transfer matrix of the inhomogeneous model is conjectured [14] to possess the doubly-degenerate eigenvalue

$$
\Theta_{n}\left(u \mid u_{1}, \ldots, u_{2 n+1}\right)=\prod_{i=1}^{2 n+1} r\left(u_{i}-u\right),
$$

where

$$
r(u)=a(u)+b(u)=\vartheta_{4}\left(0, p^{2}\right) \vartheta_{1}\left(u+\eta, p^{2}\right) \vartheta_{4}\left(u+\eta, p^{2}\right) .
$$

For the homogeneous model, where $u_{1}=\cdots=u_{2 n+1}=0$, it reduces to the eigenvalue $\Theta_{n}=$ $(a+b)^{2 n+1}$, whose existence has been rigorously established [3]. 


\section{The eigenvalue problem}

Since the transfer-matrix eigenvalue is doubly degenerate, it is convenient to lift the degeneracy by imposing the transformation property of the eigenvectors under spin reversal. We consider the following eigenvalue problem

$$
T\left(u \mid u_{1}, \ldots, u_{2 n+1}\right)|\Psi\rangle=\Theta_{n}\left(u \mid u_{1}, \ldots, u_{2 n+1}\right)|\Psi\rangle, \quad F|\Psi\rangle=(-1)^{n}|\Psi\rangle,
$$

where $|\Psi\rangle \in V^{2 n+1}$. For small $n$, it is possible to find nontrivial solutions to this system of linear equations through a direct calculation. For arbitrary $n$, however, their existence has, to our best knowledge, not been rigorously established. The following conjecture postulates this existence and characterises the corresponding space of solutions.

Conjecture 2.1 (Zinn-Justin [15]). The space of solutions to the eigenvalue problem (2.19) is spanned by a vector

$$
\left|\Psi_{n}\right\rangle=\left|\Psi_{n}\left(u_{1}, \ldots, u_{2 n+1}\right)\right\rangle \in V^{2 n+1},
$$

whose components are entire functions with respect to $u_{i}$, for each $i=1, \ldots, 2 n+1$. They are generically non-vanishing and do not have a common factor that depends on $u_{1}, \ldots, u_{2 n+1}$. Furthermore, the vector obeys the relations

$$
\begin{aligned}
\left|\Psi_{n}\left(\ldots, u_{i}+2 \pi \tau, \ldots\right)\right\rangle & =p^{-4 n} \mathrm{e}^{-2 \mathrm{i} \sum_{j=1}^{2 n+1}\left(u_{i}-u_{j}\right)}\left|\Psi_{n}\left(\ldots, u_{i}, \ldots\right)\right\rangle \\
\left|\Psi_{n}\left(\ldots, u_{i}+\pi, \ldots\right)\right\rangle & =\prod_{j=1, j \neq i}^{2 n+1} \sigma_{j}^{z}\left|\Psi_{n}\left(\ldots, u_{i}, \ldots\right)\right\rangle
\end{aligned}
$$

In this article, we assume that this conjecture holds and derive our main results from it.

\section{Properties of the eigenvector}

Conjecture 2.1 implies several properties of the vector $\left|\Psi_{n}\right\rangle$, which we frequently use in the following. We recall them here in the form of four lemmas, whose proofs can be found in [15]. The first lemma expresses the action of the $\check{R}$-matrix on the vector.

Lemma 2.2 (Exchange relations). For $n \geqslant 1$ and each $i=1, \ldots, 2 n$, we have

$$
\check{R}_{i, i+1}\left(u_{i+1}-u_{i}\right)\left|\Psi_{n}\left(\ldots, u_{i}, u_{i+1}, \ldots\right)\right\rangle=r\left(u_{i+1}-u_{i}\right)\left|\Psi_{n}\left(\ldots, u_{i+1}, u_{i}, \ldots\right)\right\rangle .
$$

We note that the compatibility of the exchange relations follows from the braid Yang-Baxter equation (2.12).

The second lemma describes the action of a local spin-flip operator on the eigenvector.

Lemma 2.3 (Local spin flips). For each $i=1, \ldots, 2 n+1$, we have

$$
\sigma_{i}^{x}\left|\Psi_{n}\left(\ldots, u_{i}, \ldots\right)\right\rangle=(-p)^{n} \mathrm{e}^{-\mathrm{i} \sum_{j=1}^{2 n+1}\left(u_{j}-u_{i}\right)}\left|\Psi_{n}\left(\ldots, u_{i}+\pi \tau, \ldots\right)\right\rangle .
$$

For the third lemma, we consider three inhomogeneity parameters $u_{i}, u_{j}, u_{k}$ with $1 \leqslant i<j<$ $k \leqslant 2 n+1$. We say that these parameters form a wheel if they can be written as $u_{i}=x, u_{j}=$ $x+2 \eta, u_{k}=x+4 \eta$, for some $x$. The so-called wheel condition asserts that the vector vanishes whenever a wheel is formed.

Lemma 2.4 (Wheel condition). For $n \geqslant 1$ and all $x$, we have

$$
\left|\Psi_{n}(\ldots, x, \ldots, x+2 \eta, \ldots, x+4 \eta, \ldots)\right\rangle=0
$$


The fourth lemma provides so-called reduction relations for the vector: Upon a specialisation of certain inhomogeneity parameters, $\left|\Psi_{n}\right\rangle$ can be expressed in terms of $\left|\Psi_{n-1}\right\rangle$. To state the reduction relations, we define for each $L \geqslant 1$ and each $1 \leqslant i \leqslant L+1$ a linear operator $\varphi_{i}: V^{L} \rightarrow V^{L+2}$. It acts on the basis vector labelled by $\boldsymbol{\alpha}=\alpha_{1} \cdots \alpha_{L}$ according to

$$
\varphi_{i}|\boldsymbol{\alpha}\rangle=\bigotimes_{j=1}^{i-1}\left|\alpha_{j}\right\rangle \otimes|s\rangle \otimes \bigotimes_{j=i}^{L}\left|\alpha_{j}\right\rangle,
$$

where

$$
|s\rangle=|\uparrow \downarrow\rangle-|\downarrow \uparrow\rangle .
$$

Lemma 2.5 (Reduction relations). For $n \geqslant 1$ and each $i=1, \ldots, 2 n$, we have

$$
\begin{aligned}
\mid \Psi_{n}\left(\ldots, u_{i-1}, u_{i}, u_{i+1}\right. & \left.\left.=u_{i}+2 \eta, u_{i+2} \ldots\right)\right\rangle \\
=C_{n} & \left(\prod_{j=1, j \neq i, i+1}^{2 n+1} \vartheta_{1}\left(u_{i}-u_{j}-2 \eta\right)\right) \varphi_{i}\left|\Psi_{n-1}\left(\ldots, u_{i-1}, u_{i+2}, \ldots\right)\right\rangle,
\end{aligned}
$$

where $C_{n}$ is independent of $u_{1}, \ldots, u_{2 n+1}$.

\section{Normalisation}

The constant $C_{n}$ in Lemma 2.5 depends on the relative normalisation of the vectors. It is not fixed by Conjecture 2.1. In the following, we choose

$$
C_{n}=1
$$

for each $n \geqslant 1$. Furthermore, we define

$$
\left|\Psi_{0}\right\rangle=|\uparrow\rangle+|\downarrow\rangle .
$$

These choices and the properties listed in Conjecture 2.1 completely fix the vector $\left|\Psi_{n}\right\rangle$ for each $n \geqslant 0$ [15]. For instance, for $n=1$ its components are

$$
\begin{aligned}
& \left(\Psi_{1}\right)_{\uparrow \uparrow \uparrow}=-\left(\Psi_{1}\right)_{\downarrow \downarrow \downarrow}=\rho \vartheta_{1}\left(u_{2}-u_{1}+\eta, p^{2}\right) \vartheta_{1}\left(u_{3}-u_{2}+\eta, p^{2}\right) \vartheta_{1}\left(u_{1}-u_{3}+\eta, p^{2}\right), \\
& \left(\Psi_{1}\right)_{\uparrow \downarrow \downarrow}=-\left(\Psi_{1}\right)_{\downarrow \uparrow \uparrow}=\rho \vartheta_{4}\left(u_{2}-u_{1}+\eta, p^{2}\right) \vartheta_{1}\left(u_{3}-u_{2}+\eta, p^{2}\right) \vartheta_{4}\left(u_{1}-u_{3}+\eta, p^{2}\right), \\
& \left(\Psi_{1}\right)_{\downarrow \uparrow \downarrow}=-\left(\Psi_{1}\right)_{\uparrow \downarrow \uparrow}=\rho \vartheta_{4}\left(u_{2}-u_{1}+\eta, p^{2}\right) \vartheta_{4}\left(u_{3}-u_{2}+\eta, p^{2}\right) \vartheta_{1}\left(u_{1}-u_{3}+\eta, p^{2}\right), \\
& \left(\Psi_{1}\right)_{\downarrow \downarrow \uparrow}=-\left(\Psi_{1}\right)_{\uparrow \uparrow \downarrow}=\rho \vartheta_{1}\left(u_{2}-u_{1}+\eta, p^{2}\right) \vartheta_{4}\left(u_{3}-u_{2}+\eta, p^{2}\right) \vartheta_{4}\left(u_{1}-u_{3}+\eta, p^{2}\right),
\end{aligned}
$$

where the overall factor is given by

$$
\rho=\frac{2}{\vartheta_{2}(0) \vartheta_{4}\left(0, p^{2}\right)}
$$

For $n \geqslant 2$, the components are considerably more complicated. An explicit formula for these components remains to be found. 


\section{$3 \quad$ Scalar products}

In this section, we compute several scalar products that involve the transfer-matrix eigenvector $\left|\Psi_{n}\right\rangle$. In Section 3.1, we define the scalar products with the help of two solutions to the boundary Yang-Baxter equation. In Section 3.2, we analyse their symmetry and analyticity properties, and show that they obey a set of reduction relations. These properties are sufficient to characterise the scalar products uniquely. In Section 3.3, we find determinant formulas in terms of the so-called elliptic Tsuchiya determinant that fulfil the same properties as the scalar products. Using uniqueness and an inductive proof technique, we show that they provide explicit expressions for the scalar products. In Section 3.4, we recall a relation between Tsuchiya determinants and a family of multivariable polynomials introduced by Zinn-Justin and Rosengren.

\subsection{Definition}

In its vector form, the boundary Yang-Baxter equation for the eight-vertex model is given by

$$
\check{R}_{1,2}(x-y) \check{R}_{2,3}(-x-y)|\chi(x)\rangle \otimes|\chi(y)\rangle=\check{R}_{3,4}(x-y) \check{R}_{2,3}(-x-y)|\chi(y)\rangle \otimes|\chi(x)\rangle,
$$

where $|\chi(x)\rangle \in V^{2}{ }^{2}$ We consider the following two solutions of this equation:

$$
\begin{aligned}
& |\chi(x)\rangle=\vartheta_{1}\left(x+\lambda, p^{2}\right) \vartheta_{4}\left(x-\lambda-2 \eta, p^{2}\right)|\uparrow \downarrow\rangle+\vartheta_{1}\left(x-\lambda-2 \eta, p^{2}\right) \vartheta_{4}\left(x+\lambda, p^{2}\right)|\downarrow \uparrow\rangle, \\
& |\bar{\chi}(x)\rangle=\vartheta_{1}\left(x+\lambda, p^{2}\right) \vartheta_{1}\left(x-\lambda-2 \eta, p^{2}\right)|\uparrow \uparrow\rangle+\vartheta_{4}\left(x-\lambda-2 \eta, p^{2}\right) \vartheta_{4}\left(x+\lambda, p^{2}\right)|\downarrow \downarrow\rangle .
\end{aligned}
$$

Here, $\lambda$ is an arbitrary parameter. Both these solutions are specialisations of the most general solution to the boundary Yang-Baxter equation for the eight-vertex model found by Inami and Konno [21]. In the following two lemmas, we list some useful properties of $|\chi(x)\rangle$ and $|\bar{\chi}(x)\rangle$. They follow from standard identities for Jacobi theta functions. It will be convenient to define

$$
g(x)=\frac{\vartheta_{4}\left(2(\eta+x), p^{2}\right)}{\vartheta_{4}\left(2(\eta-x), p^{2}\right)}, \quad \bar{g}(x)=\frac{\vartheta_{1}\left(2(\eta+x), p^{2}\right)}{\vartheta_{1}\left(2(\eta-x), p^{2}\right)} .
$$

Lemma 3.1. We have the relations

$$
\check{R}(2 x)|\chi(x)\rangle=g(x) r(2 x)|\chi(-x)\rangle, \quad \check{R}(2 x)|\bar{\chi}(x)\rangle=\bar{g}(x) r(2 x)|\bar{\chi}(-x)\rangle .
$$

Lemma 3.2. We have the matrix elements

$$
\begin{aligned}
\frac{\left(\left\langle\chi(x+2 \eta)|\otimes\langle\chi(x)|) \check{R}_{2,3}(-2(x+\eta))(|s\rangle \otimes|s\rangle)\right.\right.}{r(-2(x+\eta))} & =\frac{\vartheta_{2}(0)^{2} \vartheta_{1}(x-\lambda) \vartheta_{1}(x+\lambda+2 \eta) g(x)}{2} \\
\frac{\left(\left\langle\bar{\chi}(x+2 \eta)|\otimes\langle\bar{\chi}(x)|) \check{R}_{2,3}(-2(x+\eta))(|s\rangle \otimes|s\rangle)\right.\right.}{r(-2(x+\eta))} & =\frac{\vartheta_{2}(0)^{2} \vartheta_{1}(x-\lambda) \vartheta_{1}(x+\lambda+2 \eta) \bar{g}(x)}{2}
\end{aligned}
$$

where $|s\rangle$ is the vector defined in (2.27).

For each $n \geqslant 1$, we use the two solutions of the boundary Yang-Baxter equation to define the vectors

$$
\begin{aligned}
\left|\xi_{n}\left(x_{1}, \ldots, x_{n}\right)\right\rangle & =\left(\bigotimes_{i=1}^{n}\left|\chi\left(x_{i}\right)\right\rangle\right) \otimes|\uparrow\rangle, \\
\left|\bar{\xi}_{n}^{ \pm}\left(x_{1}, \ldots, x_{n}\right)\right\rangle & =\left(\bigotimes_{i=1}^{n}\left|\bar{\chi}\left(x_{i}\right)\right\rangle\right) \otimes(|\uparrow\rangle \pm|\downarrow\rangle) .
\end{aligned}
$$

\footnotetext{
${ }^{2}$ Traditionally, the boundary Yang-Baxter equation is presented in a matrix form. Its solutions are the so-called $K$-matrices. To each $K$-matrix $K(x)$ corresponds a solution $|\chi(x)\rangle$ of the vector form.
} 
It will also be convenient to introduce

$$
\left|\xi_{0}\right\rangle=|\uparrow\rangle, \quad\left|\bar{\xi}_{0}^{ \pm}\right\rangle=|\uparrow\rangle \pm|\downarrow\rangle .
$$

Using these vectors, we define the scalar products

$$
\begin{aligned}
& Z_{n}\left(x_{1}, \ldots, x_{n}\right)=\left\langle\xi_{n}\left(x_{1}, \ldots, x_{n}\right) \mid \Psi_{n}\left(x_{1},-x_{1}, \ldots, x_{n},-x_{n}, 0\right)\right\rangle, \\
& \bar{Z}_{n}^{ \pm}\left(x_{1}, \ldots, x_{n}\right)=\left\langle\bar{\xi}_{n}^{ \pm}\left(x_{1}, \ldots, x_{n}\right) \mid \Psi_{n}\left(x_{1},-x_{1}, \ldots, x_{n},-x_{n}, 0\right)\right\rangle .
\end{aligned}
$$

In the following, we only write out their dependence on the inhomogeneity parameters $x_{1}, \ldots, x_{n}$ if necessary.

It is straightforward to find $Z_{n}$ and $\bar{Z}_{n}^{ \pm}$for $n=0$ and $n=1$. Using (2.30), we obtain

$$
Z_{0}=1, \quad \bar{Z}_{0}^{+}=2, \quad \bar{Z}_{0}^{-}=0 .
$$

Furthermore, we use the components (2.31) and find

$$
\begin{aligned}
Z_{1} & =\frac{1}{2} \rho \vartheta_{2}(0) \vartheta_{2}(\eta+\lambda) \vartheta_{4}\left(2\left(\eta+x_{1}\right), p^{2}\right) \vartheta_{1}\left(\eta-x_{1}\right) \vartheta_{1}\left(\eta+x_{1}\right), \\
\bar{Z}_{1}^{+} & =\rho \vartheta_{4}(0) \vartheta_{4}(\eta+\lambda) \vartheta_{1}\left(2\left(\eta+x_{1}\right), p^{2}\right) \vartheta_{3}\left(\eta+x_{1}\right) \vartheta_{3}\left(\eta-x_{1}\right), \\
\bar{Z}_{1}^{-} & =\rho \vartheta_{3}(0) \vartheta_{4}(\eta+\lambda) \vartheta_{1}\left(2\left(\eta+x_{1}\right), p^{2}\right) \vartheta_{4}\left(\eta+x_{1}\right) \vartheta_{4}\left(\eta-x_{1}\right),
\end{aligned}
$$

where $\rho$ is defined in (2.32).

\subsection{Properties}

In this section, we establish the properties of $Z_{n}$ and $\bar{Z}_{n}^{ \pm}$that allow us to find them for $n \geqslant 2$. The proofs of these properties are very similar for $Z_{n}$ and $\bar{Z}_{n}^{ \pm}$. Hence, we focus on $Z_{n}$. If necessary, we indicate the modifications to be made for $\bar{Z}_{n}^{ \pm}$.

\section{Symmetry}

Proposition 3.3. For $n \geqslant 2, Z_{n}$ and $\bar{Z}_{n}^{ \pm}$are symmetric functions of $x_{1}, \ldots, x_{n}$.

Proof. We sketch the proof of the symmetry of $Z_{n}$. It is sufficient to prove that

$$
Z_{n}\left(\ldots, x_{i}, x_{i+1}, \ldots\right)=Z_{n}\left(\ldots, x_{i+1}, x_{i}, \ldots\right)
$$

for each $i=1, \ldots, n-1$. For $i=1$, we find

$$
\begin{aligned}
Z_{n}\left(x_{1}, x_{2}, \ldots\right) & =\left\langle\xi_{n}\left(x_{1}, x_{2}, \ldots\right) \mid \Psi_{n}\left(x_{1},-x_{1}, x_{2},-x_{2}, \ldots\right)\right\rangle \\
& =\frac{\left\langle\xi_{n}\left(x_{1}, x_{2}, \ldots\right)\left|\check{R}_{2,3}\left(-x_{2}-x_{1}\right) \check{R}_{3,4}\left(x_{2}-x_{1}\right)\right| \Psi_{n}\left(x_{1}, x_{2},-x_{2},-x_{1}, \ldots\right)\right\rangle}{r\left(-x_{2}-x_{1}\right) r\left(x_{2}-x_{1}\right)} \\
& =\frac{\left\langle\xi_{n}\left(x_{2}, x_{1}, \ldots\right)\left|\check{R}_{2,3}\left(-x_{2}-x_{1}\right) \check{R}_{1,2}\left(x_{2}-x_{1}\right)\right| \Psi_{n}\left(x_{1}, x_{2},-x_{2},-x_{1}, \ldots\right)\right\rangle}{r\left(-x_{2}-x_{1}\right) r\left(x_{2}-x_{1}\right)} \\
& =\left\langle\xi_{n}\left(x_{2}, x_{1}, \ldots\right) \mid \Psi_{n}\left(x_{2},-x_{2}, x_{1},-x_{1}, \ldots\right)\right\rangle=Z_{n}\left(x_{2}, x_{1}, \ldots\right) .
\end{aligned}
$$

From the first to the second line, we used Lemma 2.2. The third line is obtained by using the symmetry of the $\check{R}$-matrix and the boundary Yang-Baxter equation (3.1). The fourth line is the result of another application of Lemma 2.2. This establishes (3.14) for $i=1$. The cases where $i=2, \ldots, n-1$ are straightforward generalisations. 
Proposition 3.4. For each $i=1, \ldots, n$, we have

$$
\begin{aligned}
\vartheta_{4}\left(2\left(\eta+x_{i}\right), p^{2}\right) Z_{n}\left(\ldots,-x_{i}, \ldots\right) & =\vartheta_{4}\left(2\left(\eta-x_{i}\right), p^{2}\right) Z_{n}\left(\ldots, x_{i}, \ldots\right), \\
\vartheta_{1}\left(2\left(\eta+x_{i}\right), p^{2}\right) \bar{Z}_{n}^{ \pm}\left(\ldots,-x_{i}, \ldots\right) & =\vartheta_{1}\left(2\left(\eta-x_{i}\right), p^{2}\right) \bar{Z}_{n}^{ \pm}\left(\ldots, x_{i}, \ldots\right) .
\end{aligned}
$$

Proof. We present the proof of the first relation. By Proposition 3.3, it is sufficient to consider $i=1$. We compute

$$
\begin{aligned}
Z_{n}\left(-x_{1}, \ldots\right)=\left\langle\xi_{n}(-\right. & \left.x_{1}, \ldots\right)\left|\Psi_{n}\left(-x_{1}, x_{1}, \ldots\right)\right\rangle \\
= & \frac{\left\langle\xi_{n}\left(x_{1}, \ldots\right)\left|\check{R}_{1,2}\left(2 x_{1}\right)\right| \Psi_{n}\left(-x_{1}, x_{1}, \ldots\right)\right\rangle}{g\left(x_{1}\right) r\left(2 x_{1}\right)}=g\left(-x_{1}\right) Z_{n}\left(x_{1}, \ldots\right) .
\end{aligned}
$$

From the first to the second line, we used Lemma 3.1 and the symmetry of the $\check{R}$-matrix. The equality in the second line follows from Lemma 2.2 and the explicit expression of $g(x)$, given above.

The proof of the second relation is similar.

\section{Analyticity}

In the following, we use the concept of a theta function [22]. Let $m \geqslant 0$ be an integer. A theta function of degree $m$, nome $p$ and norm $t$ is an entire function $f$ with the pseudo-periodicity properties

$$
f(z+\pi)=(-1)^{m} f(z), \quad f(z+\pi \tau)=(-p)^{-m} \mathrm{e}^{-2 \mathrm{i}(m z-t)} f(z)
$$

Clearly, the norm $t$ is only defined modulo $\pi$. The resulting ambiguity is, however, not important for our considerations. A simple (nontrivial) example of a theta function of degree $m=1$, nome $t$ and norm $p$ is $f(z)=\vartheta_{1}(z-t)$.

Proposition 3.5. For $n \geqslant 1, Z_{n}$ and $\bar{Z}_{n}^{ \pm}$are theta functions of degree $2(n+1)$, nome $p$ and norm $\frac{\pi}{2}+\eta$ with respect to $x_{i}$ for each $i=1, \ldots, n$.

Proof. We focus on $Z_{n}$. By Proposition 3.3, it is sufficient to establish the statement for $Z_{n}$ as a function of $x_{1}$. It follows from Conjecture 2.1 and from the definition of the vector $|\chi(x)\rangle$ that $Z_{n}$ is an entire function of $x_{1}$. To establish its pseudo-periodicity properties, we note that $|\chi(x)\rangle$ obeys the relations

$$
|\chi(x+\pi)\rangle=\sigma_{1}^{z} \sigma_{1}^{z}|\chi(x)\rangle, \quad|\chi(x+\pi \tau)\rangle=-p^{-1} \mathrm{e}^{-2 \mathrm{i}(x-\eta)} \sigma_{1}^{x} \sigma_{2}^{x}|\chi(x)\rangle .
$$

Furthermore, it follows from Conjecture 2.1 and Lemma 2.3 that

$$
\begin{aligned}
\left|\Psi_{n}\left(x_{1}+\pi,-x_{1}-\pi, \ldots\right)\right\rangle & =\sigma_{1}^{z} \sigma_{2}^{z}\left|\Psi_{n}\left(x_{1},-x_{1}, \ldots\right)\right\rangle, \\
\left|\Psi_{n}\left(x_{1}+\pi \tau,-x_{1}-\pi \tau, \ldots\right)\right\rangle & =p^{-(2 n+1)} \mathrm{e}^{-2(2 n+1) \mathrm{i} x_{1}} \sigma_{1}^{x} \sigma_{2}^{x}\left|\Psi_{n}\left(x_{1},-x_{1}, \ldots\right)\right\rangle .
\end{aligned}
$$

We combine (3.20) and (3.21) to obtain

$$
Z_{n}\left(x_{1}+\pi, \ldots\right)=\left\langle\xi_{n}\left(x_{1}+\pi, \ldots\right)\left|\sigma_{1}^{z} \sigma_{2}^{z}\right| \Psi_{n}\left(x_{1},-x_{1}, \ldots\right)\right\rangle=Z_{n}\left(x_{1}, \ldots\right),
$$

and

$$
\begin{aligned}
Z_{n}\left(x_{1}+\pi \tau, \ldots\right) & =p^{-(2 n+1)} \mathrm{e}^{-2(2 n+1) \mathrm{i} x_{1}}\left\langle\xi_{n}\left(x_{1}+\pi \tau, \ldots\right)\left|\sigma_{1}^{x} \sigma_{2}^{x}\right| \Psi_{n}\left(x_{1},-x_{1}, \ldots\right)\right\rangle \\
& =p^{-2(n+1)} \mathrm{e}^{-2 \mathrm{i}\left(2(n+1) x_{1}-(\eta+\pi / 2)\right)} Z_{n}\left(x_{1}, \ldots\right) .
\end{aligned}
$$

This ends the proof for $Z_{n}$. The proof for $\bar{Z}_{n}^{ \pm}$is similar. 


\section{Zeroes and trivial factors}

In the next proposition, we identify certain trivial zeroes of the scalar products $Z_{n}$ and $\bar{Z}_{n}^{ \pm}$. It is practical to introduce the abbreviations

$$
\beta_{1}=\eta, \quad \beta_{2}=\eta+\frac{\pi}{2}, \quad \beta_{3}=\eta+\frac{\pi}{2}+\frac{\pi \tau}{2}, \quad \beta_{4}=\eta+\frac{\pi \tau}{2} .
$$

Proposition 3.6. For $n \geqslant 1$ and each $i=1, \ldots, n$, we have

$$
\begin{aligned}
& Z_{n}\left(\ldots, x_{i}=-\beta_{1}, \ldots\right)=Z_{n}\left(\ldots, x_{i}=\beta_{1}, \ldots\right)=0 \\
& Z_{n}\left(\ldots, x_{i}=-\beta_{3}, \ldots\right)=Z_{n}\left(\ldots, x_{i}=-\beta_{4}, \ldots\right)=0, \\
& \bar{Z}_{n}^{ \pm}\left(\ldots, x_{i}=-\beta_{1}, \ldots\right)=\bar{Z}_{n}^{ \pm}\left(\ldots, x_{i}=-\beta_{2}, \ldots\right)=0 .
\end{aligned}
$$

Proof. By Proposition 3.3, it is sufficient to prove the proposition for $i=1$. We focus on the proof of (3.26). We note that

$$
\begin{aligned}
Z_{n}\left(x_{1}=-\beta_{1}, \ldots\right) & =\left\langle\xi_{n}\left(\eta, x_{2}, \ldots, x_{n}\right) \mid \Psi_{n}\left(-\eta, \eta, x_{2},-x_{2}, \ldots, x_{n},-x_{n}, 0\right)\right\rangle \\
& =\left\langle\xi_{n}\left(\eta, x_{2}, \ldots, x_{n}\right)\left|\prod_{j=1}^{2 n} \sigma_{j}^{z}\right| \Psi_{n}\left(-\eta, \eta, x_{2},-x_{2}, \ldots, x_{n},-x_{n}, 3 \eta\right)\right\rangle .
\end{aligned}
$$

The first, second and last arguments of $\left|\Psi_{n}\right\rangle$ form a wheel. Lemma 2.4 implies that the vector vanishes identically. Hence, $Z_{n}\left(x_{1}=-\beta_{1}, \ldots\right)=0$. Furthermore, it follows from Proposition 3.4 that

$$
\vartheta_{4}\left(4 \eta, p^{2}\right) Z_{n}\left(x_{1}=-\beta_{1}, \ldots\right)=\vartheta_{4}\left(0, p^{2}\right) Z_{n}\left(x_{1}=\beta_{1}, \ldots\right) .
$$

Since $\vartheta_{4}\left(0, p^{2}\right)$ is non-zero, we find that $Z_{n}\left(x_{1}=\beta_{1}, \ldots\right)=0$. Hence, we obtain (3.26) with $i=1$.

Finally, we note that (3.27) and (3.28) directly follow from Proposition 3.4 and the known zeroes of the Jacobi theta functions.

We now use the knowledge of the zeroes to find trivial factors of the scalar products. To this end, we use a factorisation property of theta functions. It follows from standard complex analysis $[23,24]$ :

Lemma 3.7. Let $f$ be a theta function of degree $m \geqslant 1$, nome $p$ and norm $t$. Let $\xi$ be a complex number such that $f(\xi)=0$, then there exists a theta function $g$ of degree $m-1$, nome $p$ and norm $t-\xi$ such that

$$
f(z)=\vartheta_{1}(z-\xi) g(z) .
$$

Proposition 3.8. For $n \geqslant 1$, we have

$$
\begin{aligned}
& Z_{n}=\left(\prod_{i=1}^{n} \vartheta_{4}\left(2\left(\eta+x_{i}\right), p^{2}\right) \vartheta_{1}\left(\eta+x_{i}\right) \vartheta_{1}\left(\eta-x_{i}\right)\right) X_{n}, \\
& \bar{Z}_{n}^{ \pm}=\left(\prod_{i=1}^{n} \vartheta_{1}\left(2\left(\eta+x_{i}\right), p^{2}\right)\right) \bar{X}_{n}^{ \pm},
\end{aligned}
$$

where $X_{n}=X_{n}\left(x_{1}, \ldots, x_{n}\right)$ and $\bar{X}_{n}^{ \pm}=\bar{X}_{n}^{ \pm}\left(x_{1}, \ldots, x_{n}\right)$ are even theta functions of degree $2(n-1)$ and $2 n$, respectively, nome $p$ and norm 0 with respect to $x_{i}$ for each $i=1, \ldots, n$. For $n \geqslant 2, X_{n}$ and $\bar{X}_{n}^{ \pm}$are symmetric functions in $x_{1}, \ldots, x_{n}$. 
Proof. We present the proof of (3.32). According to Proposition 3.5, $Z_{n}$ is a theta function with respect to $x_{i}$ with degree $2(n+1)$, nome $p$ and norm $t=\pi / 2+\eta$. By Proposition 3.6 it vanishes if $x_{i}=-\beta_{1},+\beta_{1},-\beta_{4}$ and $-\beta_{3}+\pi+\pi \tau$ (where we used the pseudo-periodicity of the scalar product). Hence, we may apply Lemma 3.7 to write $Z_{n}$ as a product of the trivial factors

$$
\begin{aligned}
\vartheta_{1}\left(x_{i}+\beta_{1}\right) \vartheta_{1}\left(x_{i}-\beta_{1}\right) \vartheta_{1}\left(x_{i}+\beta_{4}\right) \vartheta_{1}\left(x_{i}+\right. & \left.\beta_{3}-\pi-\pi \tau\right) \\
& =B \vartheta_{4}\left(2\left(x_{i}+\eta\right), p^{2}\right) \vartheta_{1}\left(\eta+x_{i}\right) \vartheta_{1}\left(\eta-x_{i}\right),
\end{aligned}
$$

where $B=-\mathrm{i} p^{-1 / 2} \vartheta_{4}\left(0, p^{2}\right)$, and a theta function with respect to $x_{i}$ of degree $2(n-1)$, nome $p$ and norm $t=0$. Since this holds for each $i=1, \ldots, n$, we obtain the factorisation (3.32), absorbing a power of the constant $B$ into the definition of $X_{n}$. The symmetry of $X_{n}$ for $n \geqslant 2$ follows from Proposition 3.3 and the symmetry of the factorised expression. Moreover, it follows from Proposition 3.4 that $X_{n}$ is an even function of each $x_{i}$.

The proof of (3.33) is similar.

Henceforth, we analyse the properties of the functions $X_{n}, \bar{X}_{n}^{ \pm}$. For coherence, we define

$$
X_{0}=Z_{0}=1, \quad \bar{X}_{0}^{+}=\bar{Z}_{0}^{+}=2, \quad \bar{X}_{0}^{-}=\bar{Z}_{0}^{-}=0 .
$$

We note that for $n=1$, the expressions (3.13) lead to

$$
\begin{aligned}
X_{1} & =\frac{1}{2} \rho \vartheta_{2}(0) \vartheta_{2}(\eta+\lambda), \\
\bar{X}_{1}^{+} & =\rho \vartheta_{4}(0) \vartheta_{4}(\eta+\lambda) \vartheta_{3}\left(\eta+x_{1}\right) \vartheta_{3}\left(\eta-x_{1}\right), \\
\bar{X}_{1}^{-} & =\rho \vartheta_{3}(0) \vartheta_{3}(\eta+\lambda) \vartheta_{4}\left(\eta+x_{1}\right) \vartheta_{4}\left(\eta-x_{1}\right),
\end{aligned}
$$

where $\rho$ is defined in (2.32).

\section{Reduction relations}

The reduction relations for the vector $\left|\Psi_{n}\right\rangle$, given in Lemma 2.5, lead to reduction relations for the functions $X_{n}$ and $\bar{X}_{n}^{ \pm}$. To state them, we define

$$
\begin{aligned}
& F(x)=\frac{\vartheta_{2}(x) \vartheta_{2}(x+\eta) \vartheta_{1}(x+\lambda) \vartheta_{1}(x-\lambda+\eta)}{\vartheta_{4}\left(0, p^{2}\right)^{2}}, \\
& \bar{F}(x)=\frac{\vartheta_{3}(x) \vartheta_{3}(x+\eta) \vartheta_{4}(x) \vartheta_{4}(x+\eta) \vartheta_{1}(x-\eta)^{2} \vartheta_{1}(x+\lambda) \vartheta_{1}(x-\lambda+\eta)}{\vartheta_{4}\left(0, p^{2}\right)^{2}} .
\end{aligned}
$$

Proposition 3.9. For $n \geqslant 2$ and for each $2 \leqslant i \leqslant n$, we have the reduction relations

$$
\begin{aligned}
X_{n}\left(x_{1}=x_{i}+\eta, \ldots, x_{i}, \ldots, x_{n}\right)=F\left(x_{i}\right) \prod_{j=2, j \neq i}^{n} \vartheta_{1}\left(x_{i}-x_{j}-\eta\right)^{2} \vartheta_{1}\left(x_{i}+x_{j}-\eta\right)^{2} \\
\times X_{n-2}\left(x_{2}, \ldots, x_{i-1}, x_{i+1}, \ldots, x_{n}\right),
\end{aligned}
$$

and

$$
\begin{aligned}
\bar{X}_{n}^{ \pm}\left(x_{1}=x_{i}+\eta, \ldots, x_{i}, \ldots, x_{n}\right)=\bar{F}\left(x_{i}\right) \prod_{j=2, j \neq i}^{n} \vartheta_{1}\left(x_{i}-x_{j}-\eta\right)^{2} \vartheta_{1}\left(x_{i}+x_{j}-\eta\right)^{2} \\
\\
\times \bar{X}_{n-2}^{ \pm}\left(x_{2}, \ldots, x_{i-1}, x_{i+1}, \ldots, x_{n}\right) .
\end{aligned}
$$


Proof. We focus on the proof of the reduction relations for $X_{n}$. To this end, we abbreviate $Z_{n}^{\prime}=Z_{n}\left(x, x+2 \eta, x_{3}, \ldots, x_{n}\right)$. Using the definition of $Z_{n}$ and Lemma 2.2, we may write

$$
Z_{n}^{\prime}=\frac{\left\langle\xi_{n}(x, x+2 \eta, \ldots)\left|\check{R}_{2,3}(-2(x+\eta))\right| \Psi_{n}(x, x+2 \eta,-x,-x-2 \eta, \ldots)\right\rangle}{r(-2(x+\eta))} .
$$

The first two arguments $x, x+2 \eta$ of the vector $\left|\Psi_{n}\right\rangle$ allow us to apply the reduction relation of Lemma 2.5. We obtain

$$
\begin{aligned}
Z_{n}^{\prime}=\vartheta_{1}(2 x) \vartheta_{1}(2(x-\eta)) \vartheta_{1}(x-2 \eta) \prod_{i=3}^{n} \vartheta_{1}\left(x-x_{i}-2 \eta\right) \vartheta_{1}\left(x+x_{i}-2 \eta\right) \\
\quad \times \frac{\left\langle\xi _ { n } ( x , x + 2 \eta , \ldots ) \left|\check{R}_{2,3}(-2(x+\eta))\left(|s\rangle \otimes\left|\Psi_{n-1}(-x,-x-2 \eta, \ldots)\right\rangle\right)\right.\right.}{r(-2(x+\eta))} .
\end{aligned}
$$

Next, we use $\check{R}(-2 \eta)|s\rangle=-2 r(-2 \eta)|s\rangle$ to write

$$
\begin{aligned}
Z_{n}^{\prime}= & \vartheta_{1}(2 x) \vartheta_{1}(2(x-\eta)) \vartheta_{1}(x-2 \eta) \prod_{i=3}^{n} \vartheta_{1}\left(x-x_{i}-2 \eta\right) \vartheta_{1}\left(x+x_{i}-2 \eta\right) \\
& \times \frac{\left\langle\xi _ { n } ( x , x + 2 \eta , \ldots ) \left|\check{R}_{2,3}(-2(x+\eta)) \check{R}_{1,2}(-2 \eta)\left(|s\rangle \otimes\left|\Psi_{n-1}(-x,-x-2 \eta, \ldots)\right\rangle\right)\right.\right.}{-2 r(-2 \eta) r(-2(x+\eta))} .
\end{aligned}
$$

With the help of the boundary Yang-Baxter equation (3.1) and the symmetry of the $\check{R}$-matrix, we may write

$$
\begin{aligned}
Z_{n}^{\prime}= & \vartheta_{1}(2 x) \vartheta_{1}(2(x-\eta)) \vartheta_{1}(x-2 \eta) \prod_{i=3}^{n} \vartheta_{1}\left(x-x_{i}-2 \eta\right) \vartheta_{1}\left(x+x_{i}-2 \eta\right) \\
& \times \frac{\left\langle\xi _ { n } ( x + 2 \eta , x , \ldots ) \left|\check{R}_{2,3}(-2(x+\eta)) \check{R}_{3,4}(-2 \eta)\left(|s\rangle \otimes\left|\Psi_{n-1}(-x,-x-2 \eta, \ldots)\right\rangle\right)\right.\right.}{-2 r(-2 \eta) r(-2(x+\eta))} \\
= & \vartheta_{1}(2 x) \vartheta_{1}(2(x-\eta)) \vartheta_{1}(x-2 \eta) \prod_{i=3}^{n} \vartheta_{1}\left(x-x_{i}-2 \eta\right) \vartheta_{1}\left(x+x_{i}-2 \eta\right) \\
& \times \frac{\left\langle\xi _ { n } ( x + 2 \eta , x , \ldots ) \left|\check{R}_{2,3}(-2(x+\eta))\left(|s\rangle \otimes\left|\Psi_{n-1}(-x-2 \eta,-x, \ldots)\right\rangle\right)\right.\right.}{-2 r(-2(x+\eta))} .
\end{aligned}
$$

The last equality follows from an application of Lemma 2.2. The resulting expression suggests yet another application of Lemma 2.5. We obtain

$$
\begin{aligned}
Z_{n}^{\prime}=\vartheta_{1}(2 x) \vartheta_{1}(2(x-\eta)) \vartheta_{1}(x-2 \eta)^{2} \prod_{i=3}^{n} \vartheta_{1}\left(x-x_{i}-2 \eta\right)^{2} \vartheta_{1}\left(x+x_{i}-2 \eta\right)^{2} \\
\times \frac{\left(\left\langle\chi(x+2 \eta)|\otimes\langle\chi(x)|) \check{R}_{2,3}(-2(x+\eta))(|s\rangle \otimes|s\rangle)\right.\right.}{2 r(-2(x+\eta))} Z_{n-2}\left(x_{3}, \ldots, x_{n}\right) .
\end{aligned}
$$

The scalar product in the second line of this equality is given in Lemma 3.2. From this scalar product and from the relation between $Z_{n}$ and $X_{n}$, given in Proposition 3.8, we infer

$$
\begin{aligned}
X_{n}\left(x, x+2 \eta, x_{3}, \ldots, x_{n}\right)=F(x-\eta) \prod_{i=3}^{n} \vartheta_{1}\left(x-x_{i}-2 \eta\right)^{2} \vartheta_{1}(x+ & \left.x_{i}-2 \eta\right)^{2} \\
& \times X_{n-2}\left(x_{3}, \ldots, x_{n}\right) .
\end{aligned}
$$


Finally, we set $x=x_{2}+\eta$ and obtain the reduction relation for $X_{n}$ with $i=2$. For $i=3, \ldots, n$, it follows from the symmetry of $X_{n}$.

The proof of the reduction relations for $\bar{X}_{n}^{ \pm}$is similar.

Proposition 3.10. For each $n \geqslant 1$, we have the reduction relations

$$
\begin{aligned}
& X_{n}\left(\beta_{2}, x_{2}, \ldots, x_{n}\right)=\frac{(-1)^{n-1} \vartheta_{2}(\eta+\lambda)}{\vartheta_{4}\left(0, p^{2}\right)}\left(\prod_{i=2}^{n-1} \vartheta_{2}\left(x_{i}\right)^{2}\right) X_{n-1}\left(x_{2}, \ldots, x_{n}\right) \\
& \bar{X}_{n}^{ \pm}\left(\beta_{3}, x_{2}, \ldots, x_{n}\right)=-p^{-n / 2} \mathrm{e}^{-2 \mathrm{i} n \eta} \frac{\vartheta_{2}(\eta) \vartheta_{3}(0) \vartheta_{3}(\eta+\lambda)}{\vartheta_{4}\left(0, p^{2}\right)}\left(\prod_{i=2}^{n} \vartheta_{3}\left(x_{i}\right)^{2}\right) \bar{X}_{n-1}^{\mp}\left(x_{2}, \ldots, x_{n}\right) \\
& \bar{X}_{n}^{ \pm}\left(\beta_{4}, x_{2}, \ldots, x_{n}\right)=-p^{-n / 2} \mathrm{e}^{-2 \mathrm{i} n \eta} \frac{\vartheta_{2}(\eta) \vartheta_{4}(0) \vartheta_{4}(\eta+\lambda)}{\vartheta_{4}\left(0, p^{2}\right)}\left(\prod_{i=2}^{n} \vartheta_{4}\left(x_{i}\right)^{2}\right) \bar{X}_{n-1}^{ \pm}\left(x_{2}, \ldots, x_{n}\right)
\end{aligned}
$$

Proof. We focus on the reduction relation for $X_{n}$. To this end, we consider the vector $\left|\Psi_{n}\right\rangle$ with arguments as set in (3.10). We choose $x_{1}=-\beta_{2}$ and apply Lemma 2.5. This leads to

$$
\begin{aligned}
\mid \Psi_{n}\left(-\beta_{2}, \beta_{2}, x_{2},-x_{2}, \ldots,\right. & \left.\left.x_{n},-x_{n}\right)\right\rangle \\
& =-\vartheta_{2}(0) \prod_{i=2}^{n} \vartheta_{2}\left(x_{i}\right)^{2} \sigma_{1}^{z}|s\rangle \otimes P\left|\Psi_{n-1}\left(x_{2},-x_{2}, \ldots, x_{n},-x_{n}\right)\right\rangle,
\end{aligned}
$$

where $P$ is the spin-parity operator, defined in (2.7). We use this relation and $\sigma_{1}^{z} \sigma_{2}^{z}|\chi(x)\rangle=$ $-|\chi(x)\rangle$ to obtain

$$
Z_{n}\left(-\beta_{2}, x_{2}, \ldots, x_{n}\right)=(-1)^{n-1} \vartheta_{2}(0) \prod_{i=2}^{n} \vartheta_{2}\left(x_{i}\right)^{2}\left(\left\langle\chi\left(-\beta_{2}\right)\left|\sigma_{1}^{z}\right| s\right\rangle\right) Z_{n-1}\left(x_{2}, \ldots, x_{n}\right) .
$$

Next, we evaluate the matrix element $\left\langle\chi\left(-\beta_{2}\right)\left|\sigma_{1}^{z}\right| s\right\rangle$ with the help of

$$
\left\langle\chi(x)\left|\sigma_{1}^{z}\right| s\right\rangle=\left\langle\chi ( x ) \left|(|\uparrow \downarrow\rangle+|\downarrow \uparrow\rangle)=\vartheta_{2}(\eta+\lambda) \vartheta_{1}(x-\eta) .\right.\right.
$$

We find

$$
Z_{n}\left(-\beta_{2}, x_{2}, \ldots, x_{n}\right)=(-1)^{n-1} \vartheta_{2}(0) \vartheta_{2}(\eta) \vartheta_{2}(\eta+\lambda) \prod_{i=2}^{n} \vartheta_{2}\left(x_{i}\right)^{2} Z_{n-1}\left(x_{2}, \ldots, x_{n}\right) .
$$

We obtain (3.48) from this equality and the relation between $Z_{n}$ and $X_{n}$, given in Proposition 3.8.

To prove the two reduction relations for $\bar{X}_{n}^{ \pm}$, one needs to use Lemma 2.3 and the scalar products

$$
\begin{aligned}
\langle\bar{\chi}(x)|(|\downarrow \downarrow\rangle-|\uparrow \uparrow\rangle) & =\vartheta_{4}(\eta+\lambda) \vartheta_{3}(x-\eta), \\
\langle\bar{\chi}(x)|(|\downarrow \downarrow\rangle+|\uparrow \uparrow\rangle) & =\vartheta_{3}(\eta+\lambda) \vartheta_{4}(x-\eta) .
\end{aligned}
$$

The calculation is similar to the proof for $X_{n}$. 


\subsection{Determinants}

In this section, we present our main results for the inhomogeneous supersymmetric eight-vertex model. They provide explicit expressions for the functions $X_{n}$ and $\bar{X}_{n}^{ \pm}$, and hence for the scalar products $Z_{n}$ and $\bar{Z}_{n}^{ \pm}$. These expressions are given in terms of the so-called elliptic Tsuchiya determinant, which originally appeared as a partition function for the SOS model [24].

\section{The elliptic Tsuchiya determinant}

Let us introduce

$$
\operatorname{Rh}(x, y)=\vartheta_{1}(x-y+\eta) \vartheta_{1}(x-y-\eta) \vartheta_{1}(x+y+\eta) \vartheta_{1}(x+y-\eta) .
$$

We define $\mathbb{H}_{0}=1$ and, for each $k \geqslant 1$, the function

$$
\mathbb{H}_{2 k}\left(x_{1}, \ldots x_{k} ; x_{k+1}, \ldots, x_{2 k}\right)=\frac{\prod_{i, j=1}^{k} \mathfrak{h}\left(x_{i}, x_{j+k}\right)}{\triangle\left(x_{1}, \ldots, x_{k}\right) \triangle\left(x_{k+1}, \ldots, x_{2 k}\right)} \operatorname{det}_{i, j=1}^{k}\left(\frac{1}{\mathfrak{h}\left(x_{i}, x_{j+k}\right)}\right),
$$

where

$$
\triangle\left(x_{1}, \ldots, x_{k}\right)=\prod_{1 \leqslant i<j \leqslant k} \vartheta_{1}\left(x_{j}-x_{i}\right) \vartheta_{1}\left(x_{j}+x_{i}\right) .
$$

For $k \geqslant 1, \mathbb{H}_{2 k}\left(x_{1}, \ldots x_{k} ; x_{k+1}, \ldots, x_{2 k}\right)$ is an even theta function of degree $2(k-1)$ and norm 0 with respect to each $x_{i}, i=1, \ldots, 2 k$. Moreover, it clearly is separately symmetric in $x_{1}, \ldots, x_{k}$ and $x_{k+1}, \ldots, x_{2 k}$. Less obvious is that it is symmetric in all its variables [15]. Here, we present a simple proof based on determinant condensation [25].

Proposition 3.11. For each $k \geqslant 1, \mathbb{H}_{2 k}\left(x_{1}, \ldots x_{k} ; x_{k+1}, \ldots, x_{2 k}\right)$ is a symmetric function of $x_{1}, \ldots, x_{2 k}$.

Proof. The case $k=1$ is trivial: We have $\mathrm{H}_{2}\left(x_{1} ; x_{2}\right)=1$, which is obviously symmetric in $x_{1}$ and $x_{2}$. Therefore, we consider $k \geqslant 2$. Let $A=\left(a_{i j}\right)_{i, j=1}^{k}$ be a matrix with $a_{k k} \neq 0$. We have the condensation identity

$$
\operatorname{det} A=a_{k k}^{-(k-2)} \operatorname{det}_{i, j=1}^{k-1}\left|\begin{array}{cc}
a_{i j} & a_{i k} \\
a_{k j} & a_{k k}
\end{array}\right| .
$$

We apply this identity to the determinant in (3.58), and obtain

$$
\begin{aligned}
\mathbb{H}_{2 k}\left(x_{1}, \ldots, x_{k} ; x_{k+1}, \ldots, x_{2 k}\right)=\frac{\prod_{i, j=1}^{k-1} \mathbb{h}\left(x_{i}, x_{j+k}\right)}{\Delta\left(x_{1}, \ldots, x_{k-1}\right) \Delta\left(x_{k+1}, \ldots, x_{2 k-1}\right)} & \times \operatorname{det}_{i, j=1}^{k-1}\left(\frac{\mathbb{H}_{4}\left(x_{i}, x_{k} ; x_{j}, x_{2 k}\right)}{\overparen{h}\left(x_{i}, x_{j+k}\right)}\right) .
\end{aligned}
$$

We note that

$$
\begin{aligned}
\mathbb{H}_{4}\left(x_{i}, x_{k} ; x_{j}, x_{2 k}\right)- & H_{4}\left(x_{i}, x_{2 k} ; x_{j}, x_{k}\right) \\
& =-\frac{\vartheta_{1}(3 \eta) \vartheta_{1}\left(x_{i}-x_{j}\right) \vartheta_{1}\left(x_{i}+x_{j}\right) \vartheta_{1}\left(x_{k}-x_{2 k}\right) \vartheta_{1}\left(x_{k}+x_{2 k}\right)}{\vartheta_{1}(\eta)} .
\end{aligned}
$$

The right-hand side vanishes since $\eta=\pi / 3$. Therefore, we have

$$
\mathfrak{H}_{4}\left(x_{i}, x_{k} ; x_{j}, x_{2 k}\right)=\mathfrak{H}_{4}\left(x_{i}, x_{2 k} ; x_{j}, x_{k}\right) .
$$

Using (3.61), we conclude that $\mathrm{H}_{2 k}\left(x_{1}, \ldots, x_{k} ; x_{k+1}, \ldots, x_{2 k}\right)$ is symmetric in $x_{k}$ and $x_{2 k}$. Since it is separately symmetric in $x_{1}, \ldots, x_{k}$ and $x_{k+1}, \ldots, x_{2 k}$, it is symmetric in all its variables. 
To stress the symmetry of the function $\mathrm{H}_{2 k}\left(x_{1}, \ldots, x_{k} ; x_{k+1}, \ldots, x_{2 k}\right)$, we omit the semicolon and simply write $\mathrm{H}_{2 k}\left(x_{1}, \ldots, x_{2 k}\right)$. Finally, we note that in [15] the following reduction relation was established:

Lemma 3.12. For each $k \geqslant 1$ and each $i=2, \ldots, 2 k$, we have

$$
\begin{aligned}
\mathfrak{H}_{2 k}\left(x_{1}=x_{i}+\eta, \ldots, x_{i}, \ldots, x_{2 k}\right)= & \prod_{j=2, j \neq i}^{2 k} \vartheta_{1}\left(x_{i}-x_{j}-\eta\right) \vartheta_{1}\left(x_{i}+x_{j}-\eta\right) \\
& \times H_{2(k-1)}\left(x_{2}, \ldots, x_{i-1}, x_{i+1}, \ldots\right) .
\end{aligned}
$$

\section{The functions $X_{n}$ and $\bar{X}_{n}^{ \pm}$}

Let $m \geqslant 1$ be an integer and $z_{1}, \ldots, z_{m}, t$ be complex numbers. We say that $z_{1}, \ldots, z_{m}$ are independent if $(i) z_{i}-z_{j} \not \equiv 0(\bmod \pi, \pi \tau)$ for all $1 \leqslant i<j \leqslant m$ and $(i i) z_{1}+\cdots+z_{m}-t \not \equiv$ $0(\bmod \pi, \pi \tau)$. We have the following property of theta functions [26]:

Theorem 3.13. Let $f, g$ be theta functions of degree $m \geqslant 1$, nome $p$ and norm $t$. If there are $m$ independent complex numbers $z_{1}, \ldots, z_{m}$ such that $f\left(z_{i}\right)=g\left(z_{i}\right)$ for each $i=1, \ldots, m$, then $f=g$.

We use this theorem to find an explicit expression $X_{n}$ in terms of the elliptic Tsuchiya determinant. To this end, we define for each $k \geqslant 0$ the functions

$$
\begin{aligned}
Y_{2 k} & =\frac{(-1)^{k}}{\vartheta_{4}\left(0, p^{2}\right)^{2 k}} \mathbb{H}_{2 k}\left(x_{1}, \ldots, x_{2 k}\right) H_{2(k+1)}\left(x_{1} \ldots, x_{2 k}, \beta_{2}, \eta+\lambda\right), \\
Y_{2 k+1} & =\frac{(-1)^{k} \vartheta_{2}(\eta+\lambda)}{\vartheta_{4}\left(0, p^{2}\right)^{2 k+1}} \mathbb{H}_{2(k+1)}\left(x_{1}, \ldots, x_{2 k+1}, \beta_{2}\right) \mathfrak{H}_{2(k+1)}\left(x_{1} \ldots, x_{2 k+1}, \eta+\lambda\right) .
\end{aligned}
$$

Lemma 3.14. The function $Y_{n}$ obeys the reduction relations of $X_{n}$ given in Propositions 3.9 and 3.10 .

Proof. The proof is a straightforward calculation using Lemma 3.12.

Theorem 3.15. For each $n \geqslant 0$, we have $X_{n}=Y_{n}$.

Proof. The proof is based on a standard induction argument that goes back to Izergin and Korepin $[16,17]$. We start by examining the base cases $n=0$ and $n=1$. From the expressions given above, we find

$$
Y_{0}=1, \quad Y_{1}=\frac{\vartheta_{2}(\eta+\lambda)}{\vartheta_{4}\left(0, p^{2}\right)} .
$$

They are equal to the expressions of $X_{0}$ and $X_{1}$, given in (3.35) and (3.36), respectively.

We now make the induction hypothesis that $X_{n}=Y_{n}$ for $n=m$ and $n=m-1$, where $m \geqslant 2$ is some integer. For the induction step, we consider $X_{m+1}$ and $Y_{m+1}$ as functions of $x_{1}$. By Proposition 3.8, $X_{m+1}$ is a theta function of degree $2 m$, nome $p$ and norm 0 . The same holds for $Y_{m+1}$, by the properties of the elliptic Tsuchiya determinant. By Lemma 3.14 both functions obey the same reduction relations. For $x_{1}= \pm\left(x_{j} \pm \eta\right), j=2, \ldots, m+1$, they allow us to express $X_{m+1}$ and $Y_{m+1}$ in terms of $X_{m-1}$ and $Y_{m-1}$, respectively. Likewise, for $x_{1}= \pm \beta_{2}$, they lead to expressions of $X_{m+1}$ and $Y_{m+1}$ in terms of $X_{m}$ and $Y_{m}$ respectively. Using these expressions and the induction hypothesis, we conclude that $X_{m+1}=Y_{m+1}$ for $4 m+2$ values of $x_{1}$. We choose $x_{2}, \ldots, x_{m+1}$ so that they contain $2 m$ independent values. By Theorem 3.13, we conclude that $X_{m+1}=Y_{m+1}$, and hence $X_{n}=Y_{n}$ for $n=m+1$ and $n=m$, which ends the induction step. The theorem follows. 
Next, we provide explicit expressions for the functions $\bar{X}_{n}^{ \pm}$in terms of the elliptic Tsuchyia determinant. For each $k \geqslant 0$, we define

$$
\begin{aligned}
\bar{Y}_{2 k}^{ \pm}=\gamma_{2 k}^{ \pm} \mathrm{H}_{2(k+1)}\left(x_{1}, \ldots,\right. & \left.x_{2 k}, 0, \beta_{4}\right) \mathrm{H}_{2(k+1)}\left(x_{1}, \ldots, x_{2 k}, \eta+\lambda, \beta_{3}\right) \\
& +\delta_{2 k}^{ \pm} \uplus_{2(k+1)}\left(x_{1}, \ldots, x_{2 k}, 0, \beta_{3}\right) \mathrm{H}_{2(k+1)}\left(x_{1}, \ldots, x_{2 k}, \eta+\lambda, \beta_{4}\right),
\end{aligned}
$$

and

$$
\begin{aligned}
\bar{Y}_{2 k+1}^{ \pm}=\gamma_{2 k+1}^{ \pm} \mathrm{H}_{2(k+1)}\left(x_{1}, \ldots, x_{2 k+1}, 0\right) \mathrm{H}_{2(k+2)}\left(x_{1}, \ldots, x_{2 k+1}, \eta+\lambda, \beta_{3}, \beta_{4}\right) \\
+\delta_{2 k+1}^{ \pm} \mathbb{H}_{2(k+1)}\left(x_{1}, \ldots, x_{2 k+1}, \eta+\lambda\right) \mathbb{H}_{2(k+2)}\left(x_{1}, \ldots, x_{2 k+1}, 0, \beta_{3}, \beta_{4}\right) .
\end{aligned}
$$

Here, the coefficients $\gamma_{2 k}^{ \pm}, \gamma_{2 k+1}^{ \pm}$and $\delta_{2 k}^{ \pm}, \delta_{2 k+1}^{ \pm}$are given by

$$
\begin{aligned}
\gamma_{2 k}^{ \pm} & =\left(\frac{p \mathrm{e}^{-2 \mathrm{i} \eta}}{\vartheta_{4}\left(0, p^{2}\right)^{2}}\right)^{k} \gamma_{0}^{ \pm}, & \delta_{2 k}^{ \pm} & =\left(\frac{p \mathrm{e}^{-2 \mathrm{i} \eta}}{\vartheta_{4}\left(0, p^{2}\right)^{2}}\right)^{k} \delta_{0}^{ \pm}, \\
\gamma_{2 k+1}^{ \pm} & =\left(\frac{p \mathrm{e}^{-2 \mathrm{i} \eta}}{\vartheta_{4}\left(0, p^{2}\right)^{2}}\right)^{k} \gamma_{1}^{ \pm}, & \delta_{2 k+1}^{ \pm} & =\left(\frac{p \mathrm{e}^{-2 \mathrm{i} \eta}}{\vartheta_{4}\left(0, p^{2}\right)^{2}}\right)^{k} \delta_{1}^{ \pm},
\end{aligned}
$$

where

$$
\begin{array}{ll}
\gamma_{0}^{+}=2\left(\frac{\vartheta_{3}(0) \vartheta_{4}(\eta+\lambda)}{\vartheta_{2}(0) \vartheta_{1}(\eta+\lambda)}\right)^{2}, & \delta_{0}^{+}=-2\left(\frac{\vartheta_{4}(0) \vartheta_{3}(\eta+\lambda)}{\vartheta_{2}(0) \vartheta_{1}(\eta+\lambda)}\right)^{2}, \\
\gamma_{0}^{-}=-\frac{2 \vartheta_{3}(0) \vartheta_{4}(0) \vartheta_{3}(\eta+\lambda) \vartheta_{4}(\eta+\lambda)}{\vartheta_{2}(0)^{2} \vartheta_{1}(\eta+\lambda)^{2}}, & \delta_{0}^{-}=\frac{2 \vartheta_{3}(0) \vartheta_{4}(0) \vartheta_{3}(\eta+\lambda) \vartheta_{4}(\eta+\lambda)}{\vartheta_{2}(0)^{2} \vartheta_{1}(\eta+\lambda)^{2}},
\end{array}
$$

and

$$
\gamma_{1}^{ \pm}=-\frac{p \mathrm{e}^{-2 \mathrm{i} \eta} \vartheta_{4}(0)}{\vartheta_{4}\left(0, p^{2}\right) \vartheta_{4}(\eta+\lambda) \vartheta_{2}(\eta)} \gamma_{0}^{ \pm}, \quad \delta_{1}^{ \pm}=-\frac{p \mathrm{e}^{-2 \mathrm{i} \eta} \vartheta_{4}(\eta+\lambda)}{\vartheta_{4}\left(0, p^{2}\right) \vartheta_{4}(0) \vartheta_{2}(\eta)} \delta_{0}^{ \pm} .
$$

Lemma 3.16. The functions $\bar{Y}_{n}^{ \pm}$satisfy the reduction relations of $\bar{X}_{n}^{ \pm}$given in Propositions 3.9 and 3.10 .

Proof. The proof is a straightforward calculation using Lemma 3.12 .

Theorem 3.17. For each $n \geqslant 0$, we have $\bar{Y}_{n}^{ \pm}=\bar{X}_{n}^{ \pm}$.

Proof. As for Theorem 3.15, the proof is based on induction. Let us check the base cases $n=0$ and $n=1$, using the explicit expressions given above. For $n=0$, we find

$$
\bar{Y}_{0}^{+}=2, \quad \bar{Y}_{0}^{-}=0 .
$$

Furthermore, for $n=1$, we obtain

$$
\bar{Y}_{1}^{ \pm}=\gamma_{1}^{ \pm} \mathbb{H}_{4}\left(x_{1}, \eta+\lambda, \beta_{3}, \beta_{4}\right)+\delta_{1}^{ \pm} \mathbb{H}_{4}\left(x_{1}, 0, \beta_{3}, \beta_{4}\right) .
$$

We have [15]

$$
\mathbb{H}_{4}\left(x, y, \beta_{3}, \beta_{4}\right)=-\frac{p^{-1} \mathrm{e}^{2 \mathrm{i} \eta} \vartheta_{2}(\eta)}{\vartheta_{2}(0)}\left(\vartheta_{3}(x+\eta) \vartheta_{3}(x-\eta) \vartheta_{4}(y)^{2}+\vartheta_{4}(x+\eta) \vartheta_{4}(x-\eta) \vartheta_{3}(y)^{2}\right) .
$$


We use this expression, together with the definition of $\gamma_{1}^{ \pm}, \delta_{1}^{ \pm}$, and find

$$
\begin{aligned}
& \bar{Y}_{1}^{+}=\frac{2 \vartheta_{4}(0) \vartheta_{4}(\eta+\lambda) \vartheta_{3}\left(\eta+x_{1}\right) \vartheta_{3}\left(\eta-x_{1}\right)}{\vartheta_{2}(0) \vartheta_{4}\left(0, p^{2}\right)}, \\
& \bar{Y}_{1}^{-}=\frac{2 \vartheta_{3}(0) \vartheta_{3}(\eta+\lambda) \vartheta_{4}\left(\eta+x_{1}\right) \vartheta_{4}\left(\eta-x_{1}\right)}{\vartheta_{2}(0) \vartheta_{4}\left(0, p^{2}\right)} .
\end{aligned}
$$

We compare our findings with (3.35) and (3.36) and conclude that $\bar{Y}_{n}^{ \pm}=\bar{X}_{n}^{ \pm}$for $n=0,1$.

Next, we make the induction hypothesis that $\bar{X}_{n}^{ \pm}=\bar{Y}_{n}^{ \pm}$for $n=m$ and $n=m-1$, where $m \geqslant 2$ is some integer. For the induction step, we essentially follow the proof of Theorem 3.15. The only differences are (i) that we need to use Lemma 3.16 and (ii) that the degree of the theta functions is now $2(m+1)$. Nonetheless, we may choose $x_{2}, \ldots, x_{m+1}$ to find enough independent points for the application of Theorem 3.13 to be possible. It allows us to conclude that $\bar{X}_{m+1}^{ \pm}=\bar{Y}_{m+1}^{ \pm}$. The induction step follows, which ends the proof.

\subsection{Polynomials}

In this section, we recall the relation between the elliptic Tsuchiya determinant and a family of polynomials introduced by Zinn-Justin and Rosengren. Moreover, we discuss the properties of these polynomials that are relevant to our forthcoming analysis of the scalar products. These properties can either be found in or easily derived from [9-12,15], but we prefer to include them here for completeness.

\section{Definition and relation to the elliptic Tsuchiya determinant}

Let us introduce

$$
h\left(w, w^{\prime}\right)=1-\left(3+\zeta^{2}\right) w w^{\prime}+\left(1-\zeta^{2}\right) w w^{\prime}\left(w+w^{\prime}\right) .
$$

We define $H_{0}=1$ and, for each $k \geqslant 1$, the function ${ }^{3}$

$$
H_{2 k}\left(w_{1}, \ldots, w_{2 k}\right)=\frac{\prod_{i, j=1}^{k} h\left(w_{i}, w_{j+k}\right)}{\Delta\left(w_{1}, \ldots, w_{k}\right) \Delta\left(w_{k+1}, \ldots, w_{2 k}\right)} \operatorname{det}_{i, j=1}^{k}\left(\frac{1}{h\left(w_{i}, w_{j+k}\right)}\right),
$$

where $\Delta\left(w_{1}, \ldots, w_{m}\right)=\prod_{1 \leqslant i<j \leqslant m}\left(w_{j}-w_{i}\right)$ denotes the Vandermonde determinant in $m$ variables. Clearly, $H_{2 k}\left(w_{1}, \ldots, w_{2 k}\right)$ is a polynomial in $w_{1}, \ldots, w_{2 k}$ and $\zeta$.

In the next proposition, we recall its relation to the elliptic Tsuchiya determinant. To this end, we use the elliptic function

$$
w(x)=\frac{\vartheta_{4}\left(\eta, p^{2}\right)}{\vartheta_{4}\left(0, p^{2}\right)} \frac{\vartheta_{1}(x)^{2}}{\vartheta_{1}(x-\eta) \vartheta_{1}(x+\eta)} .
$$

Moreover, in the following we often use a parameterisation of $\zeta$ in terms of the elliptic nome $p$ :

$$
\zeta=\left(\frac{\vartheta_{1}\left(\eta, p^{2}\right)}{\vartheta_{4}\left(\eta, p^{2}\right)}\right)^{2}
$$

Lemma 3.18. Let $k \geqslant 1$ and $w_{i}=w\left(x_{i}\right)$ for each $i=1, \ldots, 2 k$. If (3.83) holds, then we have

$$
\mathrm{H}_{2 k}\left(x_{1}, \ldots, x_{2 k}\right)=f\left(x_{1}, \ldots, x_{2 k}\right) H_{2 k}\left(w_{1}, \ldots, w_{2 k}\right),
$$

\footnotetext{
${ }^{3}$ We use Zinn-Justin's notations. The polynomials $H_{2 k}\left(w_{1}, \ldots, w_{2 k}\right)$ are related to Rosengren's polynomials $T\left(x_{1}, \ldots, x_{2 k}\right)$ by a change of variables [9].
} 
where

$$
f\left(x_{1}, \ldots, x_{2 k}\right)=\left(\frac{\vartheta_{4}\left(\eta, p^{2}\right)}{\vartheta_{1}(\eta)^{2} \vartheta_{4}\left(0, p^{2}\right)}\right)^{k(k-1)} \prod_{i=1}^{2 k}\left(\vartheta_{1}\left(x_{i}+\eta\right) \vartheta_{1}\left(x_{i}-\eta\right)\right)^{k-1} .
$$

Proof. The key observations are the two relations

$$
w(x)-w(y)=-\frac{\vartheta_{4}\left(\eta, p^{2}\right) \vartheta_{1}(\eta)^{2} \vartheta_{1}(x-y) \vartheta_{1}(x+y)}{\vartheta_{4}\left(0, p^{2}\right) \vartheta_{1}(x-\eta) \vartheta_{1}(x+\eta) \vartheta_{1}(y-\eta) \vartheta_{1}(y+\eta)},
$$

and

$$
h(w(x), w(y))=\frac{\vartheta_{1}(\eta)^{4} \mathfrak{h}(x, y)}{\left(\vartheta_{1}(x-\eta) \vartheta_{1}(x+\eta) \vartheta_{1}(y-\eta) \vartheta_{1}(y+\eta)\right)^{2}} .
$$

They follow from standard identities between Jacobi theta functions. Using them together with the definition of $H_{2 k}\left(w_{1}, \ldots, w_{2 k}\right)$ and $\mathrm{H}_{2 k}\left(x_{1}, \ldots, x_{2 k}\right)$ leads to (3.84) and (3.85).

The symmetry of the elliptic Tsuchiya determinant implies that $H_{2 k}\left(w_{1}, \ldots, w_{2 k}\right)$ is a symmetric polynomial in $w_{1}, \ldots, w_{2 k}$ for $k \geqslant 1$. In the following, we use for each $k \geqslant 1$ and $j=1, \ldots, 2 k-1$ the abbreviation

$$
H_{2 k}\left(w_{1}, \ldots, w_{j}\right) \equiv H_{2 k}\left(w_{1}, \ldots, w_{j}, 0, \ldots, 0\right) .
$$

Clearly, if $j \geqslant 2$ then $H_{2 k}\left(w_{1}, \ldots, w_{j}\right)$ is a symmetric polynomial in $w_{1}, \ldots, w_{j}$.

\section{Determinant formulas}

We combine the determinant formula (3.61) for the elliptic Tsuchiya determinant with Lemma 3.18. This leads to

$$
\begin{aligned}
& H_{2 k}\left(w_{1}, \ldots, w_{2 k}\right)=\frac{\prod_{i, j=1}^{k-1} h\left(w_{i}, w_{j+k}\right)}{\Delta\left(w_{1}, \ldots, w_{k-1}\right) \Delta\left(w_{k+1}, \ldots, w_{2 k-1}\right)} \\
& \times \operatorname{det}_{i, j=1}^{k-1}\left(\frac{H_{4}\left(w_{i}, w_{k}, w_{j+k}, w_{2 k}\right)}{h\left(w_{i}, w_{j+k}\right)}\right),
\end{aligned}
$$

for each $k \geqslant 2$, where

$$
H_{4}\left(w_{1}, \ldots, w_{4}\right)=3+\zeta^{2}+\left(\zeta^{2}-1\right)\left(w_{1}+w_{2}+w_{3}+w_{4}+\left(\zeta^{2}-1\right) w_{1} w_{2} w_{3} w_{4}\right) .
$$

The determinant formula (3.89) allows us to infer several properties of the symmetric polynomial $H_{2 k}\left(w_{1}, \ldots, w_{2 k}\right)$ for $k>2$ from $k=2$. For instance, we easily find the degree of this polynomial with respect to each of its variables.

Lemma 3.19. For each $k \geqslant 1, H_{2 k}\left(w_{1}, \ldots, w_{2 k}\right)$ is a polynomial of degree $k-1$ in $w_{i}$ for each $i=1, \ldots, 2 k$. Moreover, we have

$$
\lim _{w_{2 k-1} \rightarrow \infty} w_{2 k-1}^{-(k-1)} H_{2 k}\left(w_{1}, \ldots, w_{2 k-1}, 0\right)=\left(\zeta^{2}-1\right)^{k-1} H_{2(k-1)}\left(w_{1}, \ldots, w_{2(k-1)}\right) .
$$

Furthermore, (3.89) allows us to find a useful formula for $H_{2 k}\left(w, w^{\prime}\right)$ in terms of a determinant of a matrix with polynomial entries in $w, w^{\prime}$ and $\zeta$. Indeed, from the method of divided differences [28] we obtain: 
Lemma 3.20. For each $k \geqslant 2$, we have

$$
H_{2 k}\left(w, w^{\prime}\right)=\operatorname{det}_{i, j=0}^{k-2}\left(H_{4}\left(w, w^{\prime}\right) \eta_{i, j}+\left(\zeta^{2}-1\right)\left(\eta_{i-1, j}+\eta_{i, j-1}+\left(\zeta^{2}-1\right) w w^{\prime} \eta_{i-1, j-1}\right)\right),
$$

where $\eta_{i, j}$ is a polynomial in $\zeta$ with integer coefficients, given by

$$
\eta_{i, j}=\sum_{n=\left\lceil\frac{i+j}{3}\right\rceil}^{\min (i, j)} \frac{n !\left(3+\zeta^{2}\right)^{3 n-(i+j)}\left(\zeta^{2}-1\right)^{i+j-2 n}}{(i-n) !(j-n) !(3 n-(i+j)) !}
$$

if $i, j \geqslant 0$, and $\eta_{i, j}=0$ if $i<0$ or $j<0$.

The formula given in this lemma is quite practical to explicitly compute the polynomials $H_{2 k}\left(w, w^{\prime}\right)$ with MathematicA.

\section{Bilinear identities}

The polynomials $H_{2 k}\left(w_{1}, \ldots, w_{2 k}\right)$ satisfy bilinear identities. They follow from a Plücker relation and the well-known Desnanot-Jacobi identity [25,27], applied to the determinant in (3.81).

Lemma 3.21. For each $k \geqslant 1$ and all $x, y, u, v$, we have

$$
\begin{aligned}
& (x-y) h(u, v) H_{2(k+1)}\left(w_{1}, \ldots, w_{2 k-1}, x, y, v\right) H_{2 k}\left(w_{1}, \ldots, w_{2 k-1}, u\right) \\
+ & (y-u) h(x, v) H_{2(k+1)}\left(w_{1}, \ldots, w_{2 k-1}, y, u, v\right) H_{2 k}\left(w_{1}, \ldots, w_{2 k-1}, x\right) \\
+ & (u-x) h(y, v) H_{2(k+1)}\left(w_{1}, \ldots, w_{2 k-1}, u, x, v\right) H_{2 k}\left(w_{1}, \ldots, w_{2 k-1}, y\right)=0,
\end{aligned}
$$

and

$$
\begin{aligned}
& (x-u)(y-v) H_{2(k+2)}\left(w_{1}, \ldots, w_{2 k}, x, y, u, v\right) H_{2 k}\left(w_{1}, \ldots, w_{2 k}\right) \\
= & h(x, v) h(y, u) H_{2(k+1)}\left(w_{1}, \ldots, w_{2 k}, u, v\right) H_{2(k+1)}\left(w_{1}, \ldots, w_{2 k}, x, y\right) \\
& -h(x, y) h(u, v) H_{2(k+1)}\left(w_{1}, \ldots, w_{2 k}, y, u\right) H_{2(k+1)}\left(w_{1}, \ldots, w_{2 k}, x, v\right) .
\end{aligned}
$$

\section{Fractional linear transformations}

Finally, the polynomial $H_{2 k}\left(w_{1}, \ldots, w_{2 k}\right)$ has a simple transformation behaviour under the fractional linear transformation $\zeta \rightarrow \zeta^{\prime}$, where

$$
\zeta^{\prime}=\frac{\zeta+3}{\zeta-1}
$$

It follows from the property $\left.h\left(w, w^{\prime}\right)\right|_{\zeta \rightarrow \zeta^{\prime}}=h\left(2 w /(\zeta-1), 2 w^{\prime} /(\zeta-1)\right)$ :

Lemma 3.22. For each $k \geqslant 0$, we have

$$
\left.H_{2 k}\left(w_{1}, \ldots, w_{2 k}\right)\right|_{\zeta \rightarrow \zeta^{\prime}}=\left(\frac{2}{\zeta-1}\right)^{k(k-1)} H_{2 k}\left(\frac{2 w_{1}}{\zeta-1}, \ldots, \frac{2 w_{2 k}}{\zeta-1}\right)
$$




\section{The homogeneous limit}

In this section, we consider the case where the inhomogeneity parameters take the values $u_{1}=\cdots=u_{2 n+1}=0$. This is commonly referred to as the homogeneous limit. To study the homogeneous limit of the vector $\left|\Psi_{n}\right\rangle$, it is convenient to define

$$
\left|\psi_{n}\right\rangle=\mathcal{N}_{n}\left|\Psi_{n}(0, \ldots, 0)\right\rangle, \quad\left|\bar{\psi}_{n}\right\rangle=\mathcal{N}_{n} P\left|\Psi_{n}(0, \ldots, 0)\right\rangle .
$$

Here, $\mathcal{N}_{n}$ is a normalisation factor. We choose

$$
\mathcal{N}_{n}=\frac{(-1)^{\left\lfloor\frac{n+1}{2}\right\rfloor}}{\vartheta_{1}(\eta)^{n^{2}}}\left(\frac{\vartheta_{4}\left(0, p^{2}\right)}{\vartheta_{4}\left(\eta, p^{2}\right)}\right)^{n(n+1) / 2} .
$$

We shall see below that the vectors $\left|\psi_{n}\right\rangle$ and $\left|\bar{\psi}_{n}\right\rangle$ are non-vanishing. Their definition thus implies that they are linearly independent. They constitute a basis of the eigenspace of the eigenvalue $\Theta_{n}=(a+b)^{2 n+1}$ of the transfer matrix of the homogeneous eight-vertex model [3].

The main goal of this section is to study the basis vectors $\left|\psi_{n}\right\rangle$ and $\left|\bar{\psi}_{n}\right\rangle$ with the help of the homogeneous limits of the scalar products $Z_{n}$ and $\bar{Z}_{n}^{ \pm}$. We compute these homogeneous limits in Section 4.1 and Section 4.2, respectively. This computation allows us to obtain and analyse several components of the basis vectors. In Section 4.3, we exploit the relation between the eight-vertex model and the XYZ spin chain to compute the sum of the components of the basis vectors. In Section 4.4, we compare our results to several conjectures by Bazhanov and Mangazeev, and Razumov and Stroganov.

\subsection{The homogeneous limit of $Z_{n}$}

In this section, we compute the scalar product

$$
S_{n}=\left(\left(\left\langle\uparrow \downarrow|+\mu\langle\downarrow \uparrow|)^{\otimes n} \otimes\langle\uparrow|\right)\left|\psi_{n}\right\rangle\right.\right.
$$

from the homogeneous limit of $Z_{n}$. The results of our computations naturally depend on the elliptic nome $p$. We restrict our considerations to real $0<p<1$ and state our results in terms of the variable $\zeta$, defined in (3.83), and

$$
J_{2}=-\frac{1}{2}, \quad J_{3}=\frac{1}{1+\zeta}, \quad J_{4}=\frac{1}{1-\zeta} .
$$

We have $J_{k}=w\left(\beta_{k}\right)$ for $k=2,3,4$, where $w(x)$ is defined in (3.82). We note that the restriction of the range of the elliptic nome implies that $0<\zeta<1$.

We divide the section into three parts. First, we find a closed-form expression for $S_{n}$ in terms of the polynomials defined in Section 3.4. Second, we use this expression to compute and analyse the component $\left(\psi_{n}\right)_{\uparrow \downarrow \ldots \uparrow \uparrow \uparrow}$. Third, we evaluate the scalar product $S_{n}$ in the so-called trigonometric limit.

\section{Closed-form expression}

Theorem 4.1. For each $k \geqslant 0, S_{2 k}$ and $S_{2 k+1}$ are polynomials in $\mu$ and $\zeta$, given by

$$
\begin{aligned}
S_{2 k} & =(2 \mu)^{k} H_{2 k} H_{2(k+1)}\left(J_{2}, \bar{\mu}\right), \\
S_{2 k+1} & =(2 \mu)^{k}(\mu+1) H_{2(k+1)}\left(J_{2}\right) H_{2(k+1)}(\bar{\mu}),
\end{aligned}
$$

where $\bar{\mu}=(\mu-1)^{2} /\left(\zeta^{2}-1\right) \mu$. 
Proof. The proof has two parts. In part 1, we establish the explicit formulas for the scalar products. In part 2 , we show that these expressions yield polynomials in both $\mu$ and $\zeta$.

Part 1: Explicit formulas. First, we evaluate the scalar product $Z_{n}$ for $x_{1}=\cdots=x_{n}=0$. Using (3.10), this evaluation leads to

$$
Z_{n}(0, \ldots, 0)=\left(\vartheta_{1}\left(\lambda, p^{2}\right) \vartheta_{4}\left(\lambda-\eta, p^{2}\right)\right)^{n}\left(\left(\left\langle\uparrow \downarrow|+\mu\langle\downarrow \uparrow|)^{\otimes n} \otimes\langle\uparrow|\right)\left|\Psi_{n}(0, \ldots, 0)\right\rangle,\right.\right.
$$

where

$$
\mu=\frac{\vartheta_{4}\left(\lambda, p^{2}\right) \vartheta_{1}\left(\lambda-\eta, p^{2}\right)}{\vartheta_{1}\left(\lambda, p^{2}\right) \vartheta_{4}\left(\lambda-\eta, p^{2}\right)} .
$$

We use Proposition 3.8 to write the left-hand side of (4.7) in terms of $X_{n}$. Moreover, we use (4.1) and (4.3) to rewrite its right-hand side in terms of $S_{n}$. Solving for the latter, we obtain

$$
S_{n}=\left(\frac{\vartheta_{4}\left(\eta, p^{2}\right) \vartheta_{1}(\eta)^{2}}{\vartheta_{1}\left(\lambda, p^{2}\right) \vartheta_{4}\left(\lambda-\eta, p^{2}\right)}\right)^{n} \mathcal{N}_{n} X_{n}(0, \ldots, 0) .
$$

Second, we replace $\mathcal{N}_{n}$ by its definition (4.2), and $X_{n}$ by its explicit expression in terms of the elliptic Tsuchiya determinant, given in Theorem 3.15. This step requires to consider the cases $n=2 k$ and $n=2 k+1$ separately. We focus on the case $n=2 k$, where we obtain

$$
\begin{aligned}
S_{2 k}=\frac{1}{\vartheta_{1}(\zeta)^{4 k(k-1)}\left(\vartheta_{1}\left(\lambda, p^{2}\right) \vartheta_{4}\left(\lambda-\eta, p^{2}\right)\right)^{2 k}} & \left(\frac{\vartheta_{4}\left(0, p^{2}\right)}{\vartheta_{4}\left(\eta, p^{2}\right)}\right)^{(2 k-1) k} \\
& \times \mathbb{H}_{2 k}(0, \ldots, 0) H_{2(k+1)}\left(0, \ldots, \beta_{2}, \eta+\lambda\right) .
\end{aligned}
$$

We now use Lemma 3.18 to rewrite the Tsuchiya determinants in terms of the polynomials of Section 3.4. After some algebra, we obtain

$$
S_{2 k}=\left(\frac{\vartheta_{1}(\lambda-\eta) \vartheta_{1}(\lambda) \vartheta_{2}(\eta) \vartheta_{2}(0) \vartheta_{4}\left(\eta, p^{2}\right)}{\vartheta_{1}\left(\lambda, p^{2}\right)^{2} \vartheta_{4}\left(\lambda-\eta, p^{2}\right)^{2} \vartheta_{4}\left(0, p^{2}\right)}\right)^{k} H_{2 k} H_{2(k+1)}\left(w\left(\beta_{2}\right), w(\lambda+\eta)\right) .
$$

Here, $w=w(x)$ is the elliptic function defined in (3.82). Moreover, the polynomials on the right-hand side of this equality implicitly depend on $\zeta$, which is given in term of $p$ by (3.83).

Third, we simplify (4.11) with the help of several classical identities between the Jacobi theta functions. For the prefactor, we use

$$
\vartheta_{1}(x) \vartheta_{2}(0)=2 \vartheta_{1}\left(x, p^{2}\right) \vartheta_{4}\left(x, p^{2}\right), \quad \vartheta_{2}(\eta) \vartheta_{4}\left(\eta, p^{2}\right)=2 \vartheta_{2}(0) \vartheta_{4}\left(0, p^{2}\right),
$$

and the relation (4.8). Moreover, the arguments of the polynomial are given by

$$
w\left(\beta_{2}\right)=J_{2}, \quad w(\eta+\lambda)=\bar{\mu} .
$$

Applying these identities, we obtain (4.5).

The proof of (4.6) is similar.

Part 2: Polynomial nature. Lemma 3.20 implies that both $H_{2 k}$ and $H_{2(k+1)}\left(J_{2}\right)$ are polynomials in $\zeta$. Moreover, one checks that $\mu H_{4}(w, \bar{\mu})$ is a polynomial in $w, \mu$ and $\zeta$. By Lemma 3.20, $\mu^{k} H_{2(k+1)}(w, \bar{\mu})$ is a polynomial in $w, \mu$, and $\zeta$. Hence, both $\mu^{k} H_{2(k+1)}(\bar{\mu})$ and $\mu^{k} H_{2(k+1)}\left(J_{2}, \bar{\mu}\right)$ are polynomials in $\mu$ and $\zeta$. The polynomial nature of $S_{2 k}$ and $S_{2 k+1}$ follows.

Finally, we comment on the parameterisation (4.8) used in this proof. By the properties of the Jacobi theta functions, $\mu$ is a meromorphic function of $\lambda$ for all $0<p<1$. The pole structure of this function implies that each real $\mu$ is the image of some $0<\lambda<\pi$ under this mapping. Hence, the statements of the theorem hold for all real $\mu$. By analytic continuation, they hold for all complex $\mu$. 


\section{Components}

Proposition 4.2. For each $k \geqslant 0$, we have the components

$$
\begin{aligned}
\left(\psi_{2 k}\right) \uparrow \downarrow \cdots \uparrow \downarrow \uparrow & =2^{k} H_{2 k} H_{2 k}\left(J_{2}\right), \\
\left(\psi_{2 k+1}\right)_{\uparrow \downarrow \cdots \uparrow \downarrow \uparrow} & =2^{k} H_{2 k} H_{2(k+1)}\left(J_{2}\right) .
\end{aligned}
$$

Proof. We present the proof of (4.14). For $k=0$ it is trivial. Hence, we consider $k \geqslant 1$. We have

$$
\left(\psi_{2 k}\right)_{\uparrow \downarrow \cdots \uparrow \downarrow \uparrow}=\left.S_{2 k}\right|_{\mu=0}=2^{k} H_{2 k} \lim _{\mu \rightarrow 0} \mu^{k} H_{2(k+1)}\left(J_{2}, \bar{\mu}\right) .
$$

We compute the limit on the right-hand side with the help of Lemma 3.19, and find

$$
\lim _{\mu \rightarrow 0} \mu^{k} H_{2(k+1)}\left(J_{2}, \bar{\mu}\right)=\left(\zeta^{2}-1\right)^{-k} \lim _{\bar{\mu} \rightarrow \infty} \bar{\mu}^{-k} H_{2(k+1)}\left(J_{2}, \bar{\mu}\right)=H_{2 k}\left(J_{2}\right) .
$$

This result leads to (4.14). The derivation of (4.15) is similar.

This proposition implies that the component $\left(\psi_{n}\right)_{\uparrow \downarrow \cdots \uparrow \downarrow \uparrow}$ is a polynomial in the variable $\zeta$ with integer coefficients. We now compute the coefficients of its lowest-order and highest-order term. To this end, we recall that the number of alternating sign matrices of size $n$ is given by [25]

$$
A(n)=\prod_{i=0}^{n-1} \frac{(3 i+1) !}{(n+i) !}
$$

In the following, we also frequently use the number $A_{\mathrm{V}}(2 n+1)$ of vertically-symmetric alternating sign matrices of size $2 n+1$, and the number $N_{8}(2 n)$ of cyclically-symmetric transpose complement plane partitions in a $2 n \times 2 n \times 2 n$ cube $[25,29]$ :

$$
A_{\mathrm{V}}(2 k+1)=\frac{1}{2^{k}} \prod_{i=1}^{k} \frac{(6 i-2) !(2 i-1) !}{(4 i-1) !(4 i-2) !}, \quad N_{8}(2 n)=\prod_{i=0}^{n-1} \frac{(3 i+1)(6 i) !(2 i) !}{(4 i) !(4 i+1) !} .
$$

Proposition 4.3. For each $k \geqslant 0$, we have

$$
\begin{aligned}
\left(\psi_{2 k}\right)_{\uparrow \downarrow \cdots \uparrow \downarrow \uparrow} & =A(2 k)+\cdots+2 \zeta^{k(2 k-1)}, \\
\left(\psi_{2 k+1}\right)_{\uparrow \downarrow \cdots \uparrow \downarrow \uparrow} & =A(2 k+1)+\cdots+\zeta^{k(2 k+1)},
\end{aligned}
$$

where $\cdots$ denotes intermediate powers of $\zeta$.

Proof. We compute the coefficients of the lowest-order term through the evaluation of the components at $\zeta=0$. To this end, we use [15]

$$
\left.H_{2 k}\right|_{\zeta=0}=A_{\mathrm{V}}(2 k+1),\left.\quad H_{2 k}\left(J_{2}\right)\right|_{\zeta=0}=2^{1-k} \frac{A(2 k-1)}{A_{\mathrm{V}}(2 k-1)} .
$$

Hence, we obtain

$$
\begin{aligned}
\left.\left(\psi_{2 k+1}\right)_{\uparrow \downarrow \cdots \uparrow \downarrow \uparrow}\right|_{\zeta=0} & =\left.\left.2^{k} H_{2 k}\right|_{\zeta=0} H_{2(k+1)}\left(J_{2}\right)\right|_{\zeta=0}=A(2 k+1), \\
\left.\left(\psi_{2 k}\right)_{\uparrow \downarrow \cdots \uparrow \downarrow \uparrow}\right|_{\zeta=0} & =\left.\left.2^{k} H_{2 k}\right|_{\zeta=0} H_{2 k}\left(J_{2}\right)\right|_{\zeta=0}=\frac{2 A_{\mathrm{V}}(2 k+1) A(2 k-1)}{A_{\mathrm{V}}(2 k-1)}=A(2 k),
\end{aligned}
$$

where the last equality of the second line follows from Corollary 21 of [30]. 
To find the coefficients of the highest-order terms of the components, we analyse the highestorder terms of $H_{2 k}\left(w, w^{\prime}\right)$ for fixed $w, w^{\prime}$. For $k=1$ this analysis is trivial, since $H_{2}\left(w, w^{\prime}\right)=1$. For $k \geqslant 2$, we use the determinant formula of Lemma 3.20, and find

$$
H_{2 k}\left(w, w^{\prime}\right)=\left(1+w+w^{\prime}\right)(1+w)^{k-2}\left(1+w^{\prime}\right)^{k-2} \zeta^{k(k-1)}+\ldots,
$$

where ... denotes lower-order terms in $\zeta$. Hence, we have

$$
H_{2 k}=\zeta^{k(k-1)}+\ldots, \quad H_{2 k}\left(J_{2}\right)=2^{1-k} \zeta^{k(k-1)}+\ldots
$$

Using these results, the highest-order terms of the components follow from the explicit expressions given above.

It follows from Propositions 4.2 and 4.3 that the components $\left(\psi_{n}\right)_{\uparrow \downarrow \cdots \uparrow \downarrow \uparrow}$ and $\left(\bar{\psi}_{n}\right)_{\uparrow \downarrow \cdots \uparrow \downarrow \uparrow}=$ $(-1)^{n+1}\left(\psi_{n}\right)_{\uparrow \downarrow \cdots \uparrow \downarrow \uparrow}$ do not identically vanish. Hence, the vectors $\left|\psi_{n}\right\rangle,\left|\bar{\psi}_{n}\right\rangle$ do not identically vanish, as was anticipated above. Their linear independence follows from the fact that they are, by construction, eigenvectors of the spin-reversal operator with different eigenvalues: $F\left|\psi_{n}\right\rangle=$ $(-1)^{n}\left|\psi_{n}\right\rangle, F\left|\bar{\psi}_{n}\right\rangle=(-1)^{n+1}\left|\bar{\psi}_{n}\right\rangle$.

\section{The trigonometric limit}

We now evaluate the scalar product $S_{n}$ for $\zeta \rightarrow 0$. This evaluation corresponds to the limit $p \rightarrow 0$. After a rescaling, the weights of the eight-vertex model tend, in this limit, to the trigonometric weights of the six-vertex model at $\eta=\pi / 3$. Hence, we refer to it as the trigonometric limit. The trigonometric limit of $S_{n}$ was rigorously computed in [31] with the help of contour-integral formulas for the components of the ground-state vectors of the XXZ chain at $\Delta=-1 / 2$. Here, we show that it also follows from Theorem 4.1 and a few properties of Schur functions and symplectic characters.

Let $k \geqslant 1$ and $\lambda=\left(\lambda_{1}, \ldots, \lambda_{k}\right)$ be a partition. We recall that the Schur function $s_{\lambda}$ and the symplectic character $\chi_{\lambda}$ associated with $\lambda$ are given by

$$
s_{\lambda}\left(z_{1}, \ldots, z_{k}\right)=\frac{\operatorname{det}_{i, j=1}^{k}\left(z_{i}^{\lambda_{j}+k-j}\right)}{\operatorname{det}_{i, j=1}^{k}\left(z_{i}^{k-j}\right)},
$$

and

$$
\chi_{\lambda}\left(z_{1}, \ldots, z_{m}\right)=\frac{\operatorname{det}_{i, j=1}^{k}\left(z_{i}^{\lambda_{j}+k-j+1}-z_{i}^{-\left(\lambda_{j}+k-j+1\right)}\right)}{\operatorname{det}_{i, j=1}^{k}\left(z_{i}^{k-j+1}-z_{i}^{-(k-j+1)}\right)},
$$

respectively. Hereafter, we focus on the case where $\lambda$ is given by the double-staircase partition $Y_{k}=(\lfloor(k-i) / 2\rfloor)_{i=1}^{k}$. For $\zeta=0$, the polynomial $H_{2 k}\left(w_{1}, \ldots, w_{2 k}\right)$ is related to a symplectic character associated with this partition [15]. Through the parameterisation

$$
\bar{w}(z)=\frac{(z-1)^{2}}{1+z+z^{2}}
$$

one obtains the relation

$$
\left.H_{2 k}\left(\bar{w}\left(z_{1}\right), \ldots, \bar{w}\left(z_{2 k}\right)\right)\right|_{\zeta=0}=3^{k(k-1)} \prod_{i=1}^{2 k}\left(1+z_{i}+z_{i}^{-1}\right)^{-k+1} \chi_{Y_{2 k}}\left(z_{1}, \ldots, z_{2 k}\right),
$$

for each $k \geqslant 1$. 
The next lemma provides particular factorisation properties of Schur functions associated with the double-staircase partitions into symplectic characters. It can be proven through elementary row and column operations in the involved determinants [32].

Lemma 4.4. Let $\omega=\mathrm{e}^{\mathrm{i} \pi / 3}$ and $k \geqslant 0$ be an integer, then we have

$$
\begin{aligned}
s_{Y_{4 k+2}}\left(z_{1}, \ldots, z_{2 k}, z_{1}^{-1}, \ldots, z_{2 k}^{-1}, z, 1\right)=z^{k} & \prod_{i=1}^{2 k}\left(1+z_{i}+z_{i}^{-1}\right) \\
& \times \chi_{Y_{2 k+2}}\left(z_{1}, \ldots, z_{2 k}, z, \omega\right) \chi_{Y_{2 k}}\left(z_{1}, \ldots, z_{2 k}\right),
\end{aligned}
$$

and

$$
\begin{aligned}
s_{Y_{4 k+4}}\left(z_{1}, \ldots, z_{2 k+1}, z_{1}^{-1}, \ldots, z_{2 k+1}^{-1},\right. & z, 1)=z^{k}(1+z) \prod_{i=1}^{2 k+1}\left(1+z_{i}+z_{i}^{-1}\right) \\
& \times \chi_{Y_{2 k+2}}\left(z_{1}, \ldots, z_{2 k+1}, z\right) \chi_{Y_{2 k+2}}\left(z_{1}, \ldots, z_{2 k+1}, \omega\right) .
\end{aligned}
$$

We now compute the trigonometric limit of $S_{n}$. To this end, we recall that an alternating sign matrix of size $n$ has a unique +1 in its first row. Let $A(n, k)$ denote the number of the matrices for which this +1 is in column $k$. This number is given by [25]

$$
A(n, k)=\frac{\left(\begin{array}{c}
n+k-2 \\
n-1
\end{array}\right)\left(\begin{array}{c}
2 n-1-k \\
n-1
\end{array}\right)}{\left(\begin{array}{c}
3 n-2 \\
n-1
\end{array}\right)} A(n) .
$$

Proposition 4.5. We have

$$
\left.S_{n}\right|_{\zeta=0}=\sum_{k=0}^{n} A(n+1, k+1) \mu^{k}
$$

Proof. We evaluate the expressions of Theorem 4.1 for $\zeta=0$ with the help of (4.30). This evaluation yields

$$
\begin{aligned}
\left.S_{2 k}\right|_{\zeta=0} & =3^{-k(2 k-1)}\left(\mu^{2}-\mu+1\right)^{k} \chi_{Y_{2 k}}(1, \ldots, 1) \chi_{Y_{2(k+1)}}(1, \ldots, 1, \omega, z(\mu)), \\
\left.S_{2 k+1}\right|_{\zeta=0} & =3^{-k(2 k+1)}(\mu+1)\left(\mu^{2}-\mu+1\right)^{k} \chi_{Y_{2(k+1)}}(1, \ldots, 1, \omega) \chi_{Y_{2(k+1)}}(1, \ldots, 1, z(\mu)),
\end{aligned}
$$

for $n=2 k$ and $n=2 k+1$, respectively. Here, $z(\mu)=(\omega-\mu) /(\mu \omega-1)$.

Next, we apply Lemma 4.4 and rewrite the products of symplectic characters in terms of Schur functions. For both $n=2 k$ and $n=2 k+1$, we obtain

$$
\left.S_{n}\right|_{\zeta=0}=3^{-n(n+1) / 2}(\omega(1-\omega \mu))^{n} s_{Y_{2(n+1)}}(1, \ldots, 1, z(\mu)) .
$$

Finally, we note that that the Schur function on the right-hand side is a specialisation of the partition function of a six-vertex model on an $(n+1) \times(n+1)$ square grid with domain-wall boundary conditions [33]. The model's configurations are in bijection with the set of alternating sign matrices of size $n+1$. This bijection leads to [25]

$$
s_{Y_{2(n+1)}}(1, \ldots, 1, z(\mu))=\frac{3^{n(n+1) / 2}}{(\omega(1-\omega \mu))^{n}} \sum_{k=0}^{n} A(n+1, k+1) \mu^{k} .
$$

The substitution of this expression into (4.37) ends the proof. 


\subsection{The homogeneous limit of $\bar{Z}_{n}^{ \pm}$}

In this section, we compute the scalar products

$$
\bar{S}_{n}^{ \pm}=\left((\langle\uparrow \uparrow|+\nu\langle\downarrow \downarrow|)^{\otimes n} \otimes(\langle\uparrow| \pm\langle\downarrow|)\right)\left|\psi_{n}\right\rangle
$$

from the homogeneous limit of $\bar{Z}_{n}^{ \pm}$. As for the previous section, we divide this one into three parts. First, we find closed-form expressions for $\bar{S}_{n}^{ \pm}$. Second, we use them to compute and analyse the components $\left(\psi_{n}\right)_{\downarrow \cdots \downarrow \downarrow}$ and $\left(\psi_{n}\right)_{\uparrow \ldots \uparrow \downarrow}$. Third, we evaluate the trigonometric limit of $\bar{S}_{n}^{ \pm}$.

\section{Closed-form expression}

We use the notation

$$
\bar{\nu}=\frac{(\nu-\zeta)(\nu \zeta-1)}{\left(\zeta^{2}-1\right) \nu} .
$$

Furthermore, for each $\epsilon, \epsilon^{\prime}= \pm$, we define

$$
c_{\epsilon \epsilon^{\prime}}=(\zeta+\epsilon)\left(\nu+\epsilon^{\prime}\right), \quad d_{\epsilon}=\nu(\zeta+\epsilon)^{2}, \quad \bar{d}_{\epsilon}=\zeta(\nu+\epsilon)^{2} .
$$

Theorem 4.6. For each $k \geqslant 1$, the scalar products $\bar{S}_{2 k}^{ \pm}$and $\bar{S}_{2 k+1}^{ \pm}$are polynomials in $\nu$ and $\zeta$, given by

$$
\begin{aligned}
\bar{S}_{2 k}^{+} & =\frac{\nu^{k}\left(c_{+-}^{2} H_{2(k+1)}\left(J_{4}\right) H_{2(k+1)}\left(J_{3}, \bar{\nu}\right)-c_{-+}^{2} H_{2(k+1)}\left(J_{3}\right) H_{2(k+1)}\left(J_{4}, \bar{\nu}\right)\right)}{2(\nu-\zeta)(\nu \zeta-1)}, \\
\bar{S}_{2 k}^{-} & =\frac{c_{+-} c_{-+} \nu^{k}\left(H_{2(k+1)}\left(J_{4}\right) H_{2(k+1)}\left(J_{3}, \bar{\nu}\right)-H_{2(k+1)}\left(J_{3}\right) H_{2(k+1)}\left(J_{4}, \bar{\nu}\right)\right)}{2(\nu-\zeta)(\nu \zeta-1)},
\end{aligned}
$$

and

$$
\begin{aligned}
& \bar{S}_{2 k+1}^{+}=\frac{c_{--} \nu^{k}\left(d_{+} H_{2(k+1)} H_{2(k+2)}\left(J_{3}, J_{4}, \bar{\nu}\right)-\bar{d}_{+} H_{2(k+1)}(\bar{\nu}) H_{2(k+2)}\left(J_{3}, J_{4}\right)\right)}{2 \zeta(\nu-\zeta)(\nu \zeta-1)}, \\
& \bar{S}_{2 k+1}^{-}=\frac{c_{++} \nu^{k}\left(d_{-} H_{2(k+1)} H_{2(k+2)}\left(J_{3}, J_{4}, \bar{\nu}\right)-\bar{d}_{-} H_{2(k+1)}(\bar{\nu}) H_{2(k+2)}\left(J_{3}, J_{4}\right)\right)}{2 \zeta(\nu-\zeta)(\nu \zeta-1)} .
\end{aligned}
$$

Proof. The proof is similar to the proof of Theorem 4.1. We divide it into two parts. The first part consists of establishing the explicit formulas for the scalar products. In the second part, we show that they define polynomials in $\nu$ and $\zeta$.

Part 1: Explicit formulas. First, we evaluate the scalar products $\bar{Z}_{n}^{ \pm}$for $u_{1}=\cdots=u_{2 n+1}=0$. Using (3.11), we find

$$
Z_{n}^{ \pm}(0, \ldots, 0)=\left(\vartheta_{1}\left(\lambda, p^{2}\right) \vartheta_{1}\left(\lambda-\eta, p^{2}\right)\right)^{n}\left((\langle\uparrow \uparrow|+\nu\langle\downarrow \downarrow|)^{\otimes n} \otimes(\langle\uparrow| \pm\langle\downarrow|)\right)\left|\Psi_{n}(0, \ldots, 0)\right\rangle,
$$

where

$$
\nu=\frac{\vartheta_{4}\left(\lambda-\eta, p^{2}\right) \vartheta_{4}\left(\lambda, p^{2}\right)}{\vartheta_{1}\left(\lambda-\eta, p^{2}\right) \vartheta_{1}\left(\lambda, p^{2}\right)}
$$

We use Proposition 3.8 to write the left-hand side of (4.46) in terms of $\bar{X}_{n}^{ \pm}$. Moreover, we use (4.1) and (4.39) to write the right-hand side in terms of $\bar{S}_{n}^{ \pm}$. Solving for the latter, we obtain

$$
\bar{S}_{n}^{ \pm}=\left(\frac{\vartheta_{1}\left(2 \eta, p^{2}\right)}{\vartheta_{1}\left(\lambda, p^{2}\right) \vartheta_{1}\left(\lambda-\eta, p^{2}\right)}\right)^{n} \mathcal{N}_{n} \bar{X}_{n}^{ \pm}(0, \ldots, 0)
$$


Second, we replace $\mathcal{N}_{n}$ by its definition and $\bar{X}_{n}^{ \pm}$by its explicit form in terms of the elliptic Tsuchiya determinant, given in Theorem 3.17. This step requires to consider the cases $n=2 k$ and $n=2 k+1$ separately. As in the proof of Theorem 4.1, we present the details for $n=2 k$ :

$$
\begin{aligned}
\bar{S}_{2 k}^{ \pm}=\frac{p^{k} \mathrm{e}^{-4 k \mathrm{i} \eta}}{\vartheta_{1}(\eta)^{4 k^{2}}}( & \left.\frac{\vartheta_{1}\left(\eta, p^{2}\right)}{\vartheta_{1}\left(\lambda, p^{2}\right) \vartheta_{1}\left(\lambda-\eta, p^{2}\right) \vartheta_{4}\left(0, p^{2}\right)}\right)^{2 k}\left(\frac{\vartheta_{4}\left(0, p^{2}\right)}{\vartheta_{4}\left(\eta, p^{2}\right)}\right)^{k(2 k+1)} \\
& \times\left(\gamma_{0}^{ \pm} \mathbb{H}_{2(k+1)}\left(0, \ldots, 0, \beta_{4}\right) \mathbb{H}_{2(k+1)}\left(0, \ldots, 0, w(\eta+\lambda), \beta_{3}\right)\right. \\
& \left.\quad+\delta_{0}^{ \pm} \mathbb{H}_{2(k+1)}\left(0, \ldots, 0, \beta_{3}\right) H_{2(k+1)}\left(0, \ldots, 0, w(\eta+\lambda), \beta_{4}\right)\right) .
\end{aligned}
$$

We now rewrite the elliptic Tsuchiya determinants in terms of the polynomials of Section 3.4, using Lemma 3.18, and find

$$
\begin{aligned}
\bar{S}_{2 k}^{ \pm}=\nu^{k}\left(\gamma_{0}^{ \pm} H_{2(k+1)}\left(w\left(\beta_{4}\right)\right) H_{2(k+1)}(\right. & \left.w\left(\beta_{3}\right), w(\lambda+\eta)\right) \\
& \left.+\delta_{0}^{ \pm} H_{2(k+1)}\left(w\left(\beta_{3}\right)\right) H_{2(k+1)}\left(w\left(\beta_{4}\right), w(\lambda+\eta)\right)\right) .
\end{aligned}
$$

We note that

$$
w\left(\beta_{3}\right)=J_{3}, \quad w\left(\beta_{4}\right)=J_{4}, \quad w(\lambda+\eta)=\frac{(\nu-\zeta)(\zeta \nu-1)}{\left(\zeta^{2}-1\right) \nu}=\bar{\nu}
$$

and

$$
\begin{aligned}
& \gamma_{0}^{+}=\frac{c_{+-}^{2}}{2(\nu-\zeta)(\nu \zeta-1)}, \quad \delta_{0}^{+}=-\frac{c_{-+}^{2}}{2(\nu-\zeta)(\nu \zeta-1)}, \\
& \gamma_{0}^{-}=\frac{c_{+-} c_{-+}}{2(\nu-\zeta)(\nu \zeta-1)}, \quad \delta_{0}^{-}=-\frac{c_{+-} c_{-+}}{2(\nu-\zeta)(\nu \zeta-1)} .
\end{aligned}
$$

These relations lead to the expressions for $\bar{S}_{2 k}^{ \pm}$given above. The derivation of the expressions for $\bar{S}_{2 k+1}^{ \pm}$is similar.

Part 2: Polynomial nature. We now show that $S_{n}^{ \pm}$is a polynomial in $\nu$ and $\zeta$, focussing on $n=2 k$. Using Lemma 3.20, it is straightforward to show that $H_{2(k+1)}\left(J_{i}\right)$ and $\nu^{k} H_{2(k+1)}\left(J_{i}, \bar{\nu}\right)$ are polynomials in $\nu$ and $\zeta$ for both $i=3$ and $i=4$. Hence, $\bar{S}_{2 k}^{ \pm}$is a ratio of polynomials in $\nu$ and $\zeta$. Its denominator tends to zero if and only if $\nu \rightarrow \zeta$ or $\nu \zeta \rightarrow 1$. The numerator tends to zero in these limits, too. This is sufficient to conclude that the ratio is a polynomial in both $\nu$ and $\zeta$. The case $n=2 k+1$ is similar albeit slightly more technical.

Finally, we note that this proof relies on the meromorphic parameterisation (4.47) of $\nu$ in terms of $\lambda$. Similarly to Theorem 4.1, one checks that, under this parameterisation, any real $\nu$ has a point of its preimage in $0<\lambda<\pi$. Hence, the theorem holds for all real $\nu$, and, by analytic continuation, for all complex $\nu$.

\section{Components}

Proposition 4.7. For each $k \geqslant 0$, we have

$$
\begin{aligned}
\left(\psi_{2 k}\right)_{\downarrow \cdots \downarrow \downarrow} & =\zeta^{k} H_{2 k} H_{2(k+1)}\left(J_{3}, J_{4}\right), \\
\left(\psi_{2 k+1}\right)_{\downarrow \cdots \downarrow \downarrow} & =\zeta^{k+1} H_{2(k+1)}\left(J_{3}\right) H_{2(k+1)}\left(J_{4}\right),
\end{aligned}
$$


and

$$
\begin{aligned}
\left(\psi_{2 k}\right) \uparrow \ldots \uparrow \downarrow & =\frac{1}{2} \zeta^{k}\left((1+\zeta) H_{2 k}\left(J_{3}\right) H_{2(k+1)}\left(J_{4}\right)+(1-\zeta) H_{2 k}\left(J_{4}\right) H_{2(k+1)}\left(J_{3}\right)\right), \\
\left(\psi_{2 k+1}\right) \uparrow \cdots \uparrow \downarrow & =\frac{1}{2} \zeta^{k}\left(\left(1-\zeta^{2}\right) H_{2(k+1)} H_{2(k+1)}\left(J_{3}, J_{4}\right)+H_{2 k} H_{2(k+2)}\left(J_{3}, J_{4}\right)\right) .
\end{aligned}
$$

Proof. In terms of the scalar products $\bar{S}_{n}^{ \pm}$, we have

$$
\left(\psi_{n}\right)_{\downarrow \cdots \downarrow \downarrow}=\frac{(-1)^{n}}{2}\left(\left.\bar{S}_{n}^{+}\right|_{\nu=0}+\left.\bar{S}_{n}^{-}\right|_{\nu=0}\right), \quad\left(\psi_{n}\right)_{\uparrow \cdots \uparrow \downarrow}=\frac{1}{2}\left(\left.\bar{S}_{n}^{+}\right|_{\nu=0}-\left.\bar{S}_{n}^{-}\right|_{\nu=0}\right),
$$

where we used $\left(\psi_{n}\right)_{\downarrow \cdots \downarrow \downarrow \downarrow}=(-1)^{n}\left(\psi_{n}\right)_{\uparrow \ldots \uparrow \uparrow}$ to obtain the first expression. We compute the scalar products using the explicit expressions of Theorem 4.6. With the help of Lemma 3.19, we obtain

$$
\begin{aligned}
& \left.\bar{S}_{2 k}^{+}\right|_{\nu=0}=\frac{\zeta^{k-1}}{2}\left((1+\zeta)^{2} H_{2 k}\left(J_{3}\right) H_{2(k+1)}\left(J_{4}\right)-(1-\zeta)^{2} H_{2 k}\left(J_{4}\right) H_{2(k+1)}\left(J_{3}\right)\right), \\
& \left.\bar{S}_{2 k}^{-}\right|_{\nu=0}=\frac{\zeta^{k-1}}{2}\left(1-\zeta^{2}\right)\left(H_{2 k}\left(J_{3}\right) H_{2(k+1)}\left(J_{4}\right)-H_{2 k}\left(J_{4}\right) H_{2(k+1)}\left(J_{3}\right)\right),
\end{aligned}
$$

and

$$
\begin{aligned}
& \left.\bar{S}_{2 k+1}^{+}\right|_{\nu=0}=\frac{\zeta^{k-1}(1-\zeta)}{2}\left((1+\zeta)^{2} H_{2(k+1)} H_{2(k+1)}\left(J_{3}, J_{4}\right)-H_{2 k} H_{2(k+2)}\left(J_{3}, J_{4}\right)\right), \\
& \left.\bar{S}_{2 k+1}^{-}\right|_{\nu=0}=\frac{\zeta^{k-1}(1+\zeta)}{2}\left((1-\zeta)^{2} H_{2(k+1)} H_{2(k+1)}\left(J_{3}, J_{4}\right)-H_{2 k} H_{2(k+2)}\left(J_{3}, J_{4}\right)\right) .
\end{aligned}
$$

For the components labelled by the polarised spin configuration, we thus find

$$
\begin{aligned}
\left(\psi_{2 k}\right)_{\downarrow \cdots \downarrow \downarrow} & =\frac{\zeta^{k-1}}{2}\left((\zeta+1) H_{2 k}\left(J_{3}\right) H_{2(k+1)}\left(J_{4}\right)+(\zeta-1) H_{2 k}\left(J_{4}\right) H_{2(k+1)}\left(J_{3}\right)\right), \\
\left(\psi_{2 k+1}\right)_{\downarrow \cdots \downarrow \downarrow} & =\frac{\zeta^{k-1}}{2}\left(H_{2 k} H_{2(k+2)}\left(J_{3}, J_{4}\right)-\left(1-\zeta^{2}\right) H_{2(k+1)} H_{2(k+1)}\left(J_{3}, J_{4}\right)\right),
\end{aligned}
$$

for $n=2 k$ and $n=2 k+1$, respectively. We simplify these expressions with the help of the bilinear identities of Lemma 3.21. Indeed, we rewrite the first line by using (3.94) with $w_{1}, \ldots, w_{2 k-1}=0$ and $x=J_{3}, y=J_{4}, u=v=0$. To simplify the second line, we use the identity (3.95), specialised to $w_{1}, \ldots, w_{2 k}=0$ and $x=J_{3}, y=J_{4}, u=v=0$. These simplifications lead to the expressions (4.54) and (4.55).

For the components labelled by the almost-polarised spin configuration, the substitution yields (4.56) and (4.57).

It follows that the components $\left(\psi_{n}\right)_{\downarrow \cdots \downarrow \downarrow \downarrow}$ and $\left(\psi_{n}\right)_{\uparrow \ldots \uparrow \downarrow}$ are polynomials in $\zeta$ with integer coefficients. In the next proposition, we provide the coefficients of their lowest-order and highestorder terms. They follow from the evaluations [15]

$$
\left.H_{2(k+1)}\left(J_{3}, J_{4}\right)\right|_{\zeta=0}=A_{\mathrm{V}}(2 k+1),\left.\quad H_{2 k}\left(J_{3}\right)\right|_{\zeta=0}=\left.H_{2 k}\left(J_{4}\right)\right|_{\zeta=0}=N_{8}(2 k),
$$

and from Lemma 3.20, respectively.

Proposition 4.8. For each $k \geqslant 0$, we have

$$
\begin{aligned}
\left(\psi_{2 k}\right)_{\downarrow \cdots \downarrow \downarrow} & =A_{\mathrm{V}}(2 k+1)^{2} \zeta^{k}+\cdots+\zeta^{k(2 k+1)}, \\
\left(\psi_{2 k+1}\right)_{\downarrow \cdots \downarrow \downarrow} & =N_{8}(2 k+2)^{2} \zeta^{k+1}+\cdots+\zeta^{(k+1)(2 k+1)},
\end{aligned}
$$


and, where $\cdots$ denotes intermediate powers of $\zeta$, and

$$
\begin{aligned}
\left(\psi_{2 k}\right)_{\uparrow \cdots \uparrow \downarrow} & =N_{8}(2 k) N_{8}(2 k+2) \zeta^{k}+\cdots, \\
\left(\psi_{2 k+1}\right)_{\uparrow \ldots \uparrow \downarrow} & =A_{\mathrm{V}}(2 k+1) A_{\mathrm{V}}(2 k+3) \zeta^{k}+\cdots,
\end{aligned}
$$

where $\cdots$ denotes higher powers of $\zeta$.

The coefficient of the highest-order term of $\left(\psi_{n}\right)_{\uparrow \ldots \uparrow \downarrow}$ is rather difficult to compute. The reason is a nontrivial cancellation between several of the leading powers of the two terms on the right-hand sides of (4.56) and (4.57). For small $n$, we observe that the coefficient of the highest-order term is given by the $n$-th Catalan number $C(n)=\frac{1}{n+1}\left(\begin{array}{c}2 n \\ n\end{array}\right)$, but we have no proof for arbitrary $n$.

\section{The trigonometric limit}

Finally, we compute the trigonometric limit of $\bar{S}_{n}^{ \pm}$. It yields simple polynomials in $\nu$, in sharp contrast to the trigonometric limit of $S_{n}$ discussed above. Despite their simplicity, we have not found them in the literature on the XXZ spin chain at $\Delta=-1 / 2$.

Proposition 4.9. For each $k \geqslant 0$, we have

$$
\left.\bar{S}_{2 k}^{+}\right|_{\zeta=0}=2 A_{\mathrm{V}}(2 k+1) N_{8}(2(k+1)) \nu^{k},\left.\quad \bar{S}_{2 k}^{-}\right|_{\zeta=0}=0
$$

and

$$
\left.\bar{S}_{2 k+1}^{ \pm}\right|_{\zeta=0}=-A_{\mathrm{V}}(2 k+3) N_{8}(2(k+1))(\nu \mp 1) \nu^{k} .
$$

Proof. We use (4.65) to evaluate the closed-form expressions found in Theorem 4.6 for $\zeta=0$. The evaluation of $\bar{S}_{2 k}^{ \pm}$is straightforward and leads to (4.70). The evaluation of $\bar{S}_{2 k+1}^{ \pm}$leads, however, to a singular expression. To compute it, we use the bilinear identity (3.94) of Lemma 3.21 with $w_{1}=\cdots=w_{2 k-1}=0$. Setting $x=J_{3}, y=J_{4}, u=\bar{\nu}, v=0$, we obtain

$$
\begin{aligned}
H_{2(k+1)}\left(J_{3}, J_{4}\right) H_{2 k}(\bar{\nu})=\frac{1}{2 \nu}\left(\left(1+(1-\zeta) \nu+\nu^{2}\right) H_{2(k+1)}\left(J_{4}, \bar{\nu}\right) H_{2 k}\left(J_{3}\right)\right. \\
\left.-\left(1-(1+\zeta) \nu+\nu^{2}\right) H_{2(k+1)}\left(J_{3}, \bar{\nu}\right) H_{2 k}\left(J_{4}\right)\right) .
\end{aligned}
$$

Likewise, setting $x=J_{3}, y=J_{4}, u=0, v=\bar{\nu}$, we find

$$
\begin{array}{r}
H_{2(k+1)}\left(J_{3}, J_{4}, \bar{\nu}\right) H_{2 k}=\frac{\zeta}{2 \nu^{2}\left(\zeta^{2}-1\right)}\left((\nu-1)^{2}\left(1+(1-\zeta) \nu+\nu^{2}\right) H_{2(k+1)}\left(J_{3}, \bar{\nu}\right) H_{2 k}\left(J_{4}\right)\right. \\
\left.-(\nu+1)^{2}\left(1-(1+\zeta) \nu+\nu^{2}\right) H_{2(k+1)}\left(J_{4}, \bar{\nu}\right) H_{2 k}\left(J_{3}\right)\right) .
\end{array}
$$

Applying these relations allows us to simplify the scalar products to

$$
\begin{aligned}
& \bar{S}_{2 k+1}^{+}=\frac{\nu^{k-1}(\nu-1)}{2}\left(\left(1+\nu^{2}\right) H_{2(k+1)}\left(J_{4}\right) H_{2(k+2)}\left(J_{3}, \bar{\nu}\right)\right. \\
&\left.-(1+\nu)^{2} H_{2(k+1)}\left(J_{3}\right) H_{2(k+2)}\left(J_{4}, \bar{\nu}\right)\right),
\end{aligned}
$$

and

$$
\begin{aligned}
\bar{S}_{2 k+1}^{-}=\frac{\nu^{k-1}(\nu+1)}{2}\left((1-\nu)^{2} H_{2(k+1)}\left(J_{4}\right) H_{2(k+2)}\left(J_{3}, \bar{\nu}\right)\right. \\
\left.-\left(1+\nu^{2}\right) H_{2(k+1)}\left(J_{3}\right) H_{2(k+2)}\left(J_{4}, \bar{\nu}\right)\right) .
\end{aligned}
$$

These expressions are non-singular for $\zeta=0$. We evaluate them with the help of (4.65), which leads to (4.71). 


\subsection{The sums of components}

In this section, we compute the sum of the components of the vectors $\left|\psi_{n}\right\rangle$ and $\left|\bar{\psi}_{n}\right\rangle$, given by

$$
\Sigma_{n}=\sum_{\boldsymbol{\alpha}}\left(\psi_{n}\right)_{\boldsymbol{\alpha}}, \quad \bar{\Sigma}_{n}=\sum_{\boldsymbol{\alpha}}\left(\bar{\psi}_{n}\right)_{\boldsymbol{\alpha}}
$$

respectively. It is possible to find closed-form expressions for these sums from the homogeneous limit of a scalar product for the inhomogeneous supersymmetric eight-vertex model. To find these scalar products, one needs to follow the strategy of Section 3, but with a different solution to the boundary Yang-Baxter equation [34]. Here, we present a different approach that exploits the relation between the eight-vertex model and the XYZ spin chain.

\section{XYZ spin chain and parameter range}

Let us define the XYZ Hamiltonian

$$
H=-\frac{1}{2} \sum_{i=1}^{L} J_{4} \sigma_{i}^{x} \sigma_{i+1}^{x}+J_{3} \sigma_{i}^{y} \sigma_{i+1}^{y}+J_{2} \sigma_{i}^{z} \sigma_{i+1}^{z},
$$

whose coupling constants are given in (4.4). Furthermore, we introduce

$$
E_{0}=-\frac{(2 n+1)\left(3+\zeta^{2}\right)}{4\left(1-\zeta^{2}\right)}
$$

The vectors $\left|\psi_{n}\right\rangle$ and $\left|\bar{\psi}_{n}\right\rangle$ span the one-dimensional spaces of solutions of the eigenvalue problems

$$
\begin{array}{ll}
H|\psi\rangle=E_{0}|\psi\rangle, & F|\psi\rangle=(-1)^{n}|\psi\rangle, \\
H|\psi\rangle=E_{0}|\psi\rangle, & F|\psi\rangle=(-1)^{n+1}|\psi\rangle,
\end{array}
$$

where $|\psi\rangle \in V^{2 n+1}$, respectively [3]. We note that the Hamiltonian's eigenvalue $E_{0}$ is doubly degenerate. For $0<\zeta<1$ (and even $-1<\zeta<1$ ), it is its ground-state eigenvalue.

We now use the connection to the spin chain to characterise the vectors as functions of $\zeta$ :

Lemma 4.10. The vectors $\left|\psi_{n}\right\rangle$ and $\left|\bar{\psi}_{n}\right\rangle$ are rational functions of $\zeta$.

Proof. The space of the solution of the eigenvalue problem (4.79) is one-dimensional. Hence, if one fixes one component of $|\psi\rangle$ then all other components follow from basic linear algebra. For the solution $\left|\psi_{n}\right\rangle$, we have shown in Propositions 4.2 and 4.3 that the component $\left(\psi_{n}\right)_{\uparrow \uparrow \downarrow} \ldots \uparrow \downarrow \uparrow$ is a non-vanishing polynomial in $\zeta$. Since both $H$ and $E_{0}$ are rational functions of $\zeta$, all the components of $\left|\psi_{n}\right\rangle$ are therefore rational in $\zeta$. The rationality of $\left|\bar{\psi}_{n}\right\rangle$ follows from the relation $\left|\bar{\psi}_{n}\right\rangle=P\left|\psi_{n}\right\rangle$.

This lemma allows us to consider the vectors $\left|\psi_{n}\right\rangle$ and $\left|\bar{\psi}_{n}\right\rangle$ not only as rational functions on the interval $0<\zeta<1$ but on the the real line, forgetting about the initial parameterisation (3.83). We adopt this point of view in the following and note that the vectors span the eigenspace of $E_{0}$ for all $\zeta$. The following lemma gives the behaviour of $\left|\psi_{n}\right\rangle$ for large $\zeta$ :

Lemma 4.11. We have $\left|\psi_{n}\right\rangle=\zeta^{n(n+1) / 2}\left(|\downarrow \cdots \downarrow\rangle+(-1)^{n}|\uparrow \cdots \uparrow\rangle+o(1)\right)$ as $\zeta \rightarrow \infty$.

Proof. For $\zeta \rightarrow \infty$, the eigenvalue problem (4.79) becomes

$$
\frac{1}{4}\left(\sum_{i=1}^{2 n+1} \sigma_{i}^{z} \sigma_{i+1}^{z}\right)|\psi\rangle=\frac{2 n+1}{4}|\psi\rangle, \quad F|\psi\rangle=(-1)^{n}|\psi\rangle .
$$


The space of its solutions is spanned by $|\psi\rangle=|\downarrow \cdots \downarrow\rangle+(-1)^{n}|\uparrow \cdots \uparrow\rangle$. By Lemma 4.10, $\left|\psi_{n}\right\rangle$ is a rational function of $\zeta$. We conclude that there are an integer $d_{n}$ and a complex number $a_{n}$ such that

$$
\left|\psi_{n}\right\rangle=a_{n} \zeta^{d_{n}}\left(|\downarrow \cdots \downarrow\rangle+(-1)^{n}|\uparrow \cdots \uparrow\rangle+o(1)\right),
$$

It follows from Proposition 4.8 that $a_{n}=1$ and $d_{n}=n(n+1) / 2$.

\section{The sums of components}

We now compute $\Sigma_{n}$ and $\bar{\Sigma}_{n}$. Using the spin-reversal properties $F\left|\psi_{n}\right\rangle=(-1)^{n}\left|\psi_{n}\right\rangle$ and $F\left|\bar{\psi}_{n}\right\rangle=(-1)^{n+1}\left|\bar{\psi}_{n}\right\rangle$, we find the trivial results

$$
\Sigma_{2 k+1}=0, \quad \bar{\Sigma}_{2 k}=0
$$

for each $k \geqslant 0$. We are going to show that $\Sigma_{2 k}$ and $\bar{\Sigma}_{2 k+1}$ are, however, quite nontrivial. To this end, it will be useful to write them as follows:

$$
\Sigma_{n}=2^{n+1 / 2}\left\langle\uparrow \cdots \uparrow|U| \psi_{n}\right\rangle, \quad \bar{\Sigma}_{n}=2^{n+1 / 2}\left\langle\downarrow \cdots \downarrow|U| \psi_{n}\right\rangle .
$$

Here, $U=2^{-L / 2} \prod_{j=1}^{L}\left(1+\mathrm{i} \sigma_{j}^{y}\right)$ is an orthogonal operator on $V^{L}$. In the next lemma, we compute the action of $U$ on the vector $\left|\psi_{n}\right\rangle=\left|\psi_{n}(\zeta)\right\rangle$.

Lemma 4.12. For each $n \geqslant 0$, we have

$$
U\left|\psi_{n}(\zeta)\right\rangle=B_{n}(\zeta)\left(\left|\psi_{n}\left(\zeta^{\prime}\right)\right\rangle+(-1)^{n+1}\left|\bar{\psi}_{n}\left(\zeta^{\prime}\right)\right\rangle\right)
$$

where $\zeta^{\prime}=(\zeta+3) /(\zeta-1)$, and

$$
B_{n}(\zeta)^{2}=\frac{\left\|\psi_{n}(\zeta)\right\|^{2}}{2\left\|\psi_{n}\left(\zeta^{\prime}\right)\right\|^{2}}
$$

Proof. First, we write $H=H(\zeta)$ and $E_{0}=E_{0}(\zeta)$ for the XYZ Hamiltonian (4.77) and its special eigenvalue (4.78). One checks that they satisfy the relations

$$
H\left(\zeta^{\prime}\right) U=\left(\frac{\zeta-1}{2}\right) U H(\zeta), \quad E_{0}\left(\zeta^{\prime}\right)=\left(\frac{\zeta-1}{2}\right) E_{0}(\zeta)
$$

It follows from these relations that $U\left|\psi_{n}(\zeta)\right\rangle$ is an eigenvector of the Hamiltonian $H\left(\zeta^{\prime}\right)$ associated to the eigenvalue $E_{0}\left(\zeta^{\prime}\right)$. Since the corresponding eigenspace is spanned by $\left|\psi_{n}\left(\zeta^{\prime}\right)\right\rangle$ and $\left|\bar{\psi}_{n}\left(\zeta^{\prime}\right)\right\rangle$, we may write

$$
U\left|\psi_{n}(\zeta)\right\rangle=B_{n}(\zeta)\left|\psi_{n}\left(\zeta^{\prime}\right)\right\rangle+\bar{B}_{n}(\zeta)\left|\bar{\psi}_{n}\left(\zeta^{\prime}\right)\right\rangle
$$

where $B_{n}(\zeta), \bar{B}_{n}(\zeta)$ are coefficients.

Second, we compute the scalar product of both sides of this equality with the basis vectors $|\uparrow \cdots \uparrow\rangle$ and $|\downarrow \cdots \downarrow\rangle$. Using (4.84), we find

$$
\begin{aligned}
& \Sigma_{n}=2^{n+1 / 2}\left(B_{n}(\zeta) \psi_{n}\left(\zeta^{\prime}\right)_{\uparrow \ldots \uparrow}+\bar{B}_{n}(\zeta) \bar{\psi}_{n}\left(\zeta^{\prime}\right)_{\uparrow \ldots \uparrow}\right)=2^{n+1 / 2}\left(B_{n}(\zeta)-\bar{B}_{n}(\zeta)\right) \psi_{n}\left(\zeta^{\prime}\right)_{\uparrow \ldots \uparrow}, \\
& \bar{\Sigma}_{n}=2^{n+1 / 2}\left(B_{n}(\zeta) \psi_{n}\left(\zeta^{\prime}\right)_{\uparrow \ldots \uparrow}+\bar{B}_{n}(\zeta) \bar{\psi}_{n}\left(\zeta^{\prime}\right)_{\uparrow \ldots \uparrow}\right)=2^{n+1 / 2}\left(B_{n}(\zeta)+\bar{B}_{n}(\zeta)\right) \psi_{n}\left(\zeta^{\prime}\right)_{\downarrow \cdots \downarrow}
\end{aligned}
$$

Here, we applied the relations $\left(\bar{\psi}_{n}\right)_{\uparrow \ldots \uparrow}=-\left(\psi_{n}\right)_{\uparrow \ldots \uparrow},\left(\bar{\psi}_{n}\right)_{\downarrow \cdots \downarrow}=\left(\psi_{n}\right)_{\downarrow \cdots \downarrow}$, which straightforwardly follow from $\left|\bar{\psi}_{n}\right\rangle=P\left|\psi_{n}\right\rangle$. We now combine (4.83) with (4.89), and use the fact that the components labelled by the polarised spin configurations do not identically vanish (which follows from Proposition 4.8). This leads to

$$
\bar{B}_{2 k}(\zeta)=-B_{2 k}(\zeta), \quad \bar{B}_{2 k+1}(\zeta)=B_{2 k+1}(\zeta)
$$


for each $k \geqslant 0$. Hence, we obtain (4.85).

Third, we compute the scalar product of each side of (4.85) with itself. Using the fact that $U$ is orthogonal, as well as $\left\langle\bar{\psi}_{n}\left(\zeta^{\prime}\right) \mid \psi_{n}\left(\zeta^{\prime}\right)\right\rangle=0$ and $\left\|\bar{\psi}_{n}\left(\zeta^{\prime}\right)\right\|^{2}=\left\|\psi_{n}\left(\zeta^{\prime}\right)\right\|^{2}$, we obtain (4.86).

It is clear from this lemma why we need the continuation of $\left|\psi_{n}\right\rangle$ to values of $\zeta$ outside the range $0<\zeta<1$. Indeed, if $\zeta$ is in this range then $-\infty<\zeta^{\prime}<-3$. Moreover, the lemma shows that to find an explicit formula for the sums of components we need the square norm of the ground-state vector $\left|\psi_{n}\right\rangle$. One of the main results of [15] are the expressions

$$
\begin{aligned}
\left\|\psi_{2 k}\right\|^{2} & =2^{2 k+1} H_{2 k} H_{2(k+1)}\left(J_{2}, J_{3}\right) H_{2(k+1)}\left(J_{3}, J_{4}\right) H_{2(k+1)}\left(J_{2}, J_{4}\right), \\
\left\|\psi_{2 k+1}\right\|^{2} & =2^{2(k+1)} H_{2(k+1)}\left(J_{2}\right) H_{2(k+1)}\left(J_{3}\right) H_{2(k+1)}\left(J_{4}\right) H_{2(k+2)}\left(J_{2}, J_{3}, J_{4}\right),
\end{aligned}
$$

for each $k \geqslant 0$. We use them to obtain the following result:

Proposition 4.13. For each $k \geqslant 0$, we have

$$
\begin{aligned}
\Sigma_{2 k} & =2^{k+1}(\zeta+3)^{k} H_{2 k} H_{2(k+1)}\left(J_{2}, J_{3}\right), \\
\bar{\Sigma}_{2 k+1} & =2^{k+1}(\zeta+3)^{k+1} H_{2(k+1)}\left(J_{2}\right) H_{2(k+1)}\left(J_{3}\right) .
\end{aligned}
$$

Proof. The scalar product of (4.85) and the basis vectors $|\uparrow \cdots \uparrow\rangle$ and $|\downarrow \cdots \downarrow\rangle$ implies

$$
\Sigma_{2 k}=2^{2 k+3 / 2} B_{2 k}(\zeta) \psi_{2 k}\left(\zeta^{\prime}\right)_{\downarrow \cdots \downarrow}, \quad \bar{\Sigma}_{2 k+1}=2^{2 k+5 / 2} B_{2 k+1}(\zeta) \psi_{2 k+1}\left(\zeta^{\prime}\right)_{\downarrow \cdots \downarrow} .
$$

To find these two expressions, we used $\left(\bar{\psi}_{n}\right)_{\uparrow \ldots \uparrow}=-\left(\psi_{n}\right)_{\uparrow \ldots \uparrow},\left(\bar{\psi}_{n}\right)_{\downarrow \cdots \downarrow}=\left(\psi_{n}\right)_{\downarrow \cdots \downarrow}$, as well as $\left(\psi_{2 k}\right)_{\uparrow \ldots \uparrow}=\left(\psi_{2 k}\right)_{\downarrow \cdots \downarrow}$. We compute the coefficients $B_{2 k}(\zeta)$ and $B_{2 k+1}(\zeta)$ in these expressions by applying the transformation property of Lemma 3.22 to the square norms (4.91). We find

$$
B_{2 k}(\zeta)=\frac{b_{2 k}}{2^{1 / 2}}\left(\frac{\zeta-1}{2}\right)^{k(2 k+1)}, \quad B_{2 k+1}(\zeta)=\frac{b_{2 k+1}}{2^{1 / 2}}\left(\frac{\zeta-1}{2}\right)^{(k+1)(2 k+1)},
$$

where $b_{2 k}, b_{2 k+1}= \pm 1$ are signs that we fix below. Moreover, using Lemma 3.22 and Proposition 4.7 , we obtain

$$
\begin{aligned}
\psi_{2 k}\left(\zeta^{\prime}\right)_{\downarrow \cdots \downarrow} & =\left(\frac{\zeta+3}{\zeta-1}\right)^{k}\left(\frac{2}{\zeta-1}\right)^{2 k^{2}} H_{2 k} H_{2(k+1)}\left(J_{2}, J_{3}\right), \\
\psi_{2 k+1}\left(\zeta^{\prime}\right)_{\downarrow \cdots \downarrow} & =\left(\frac{\zeta+3}{\zeta-1}\right)^{k+1}\left(\frac{2}{\zeta-1}\right)^{2 k(k+1)} H_{2 k} H_{2(k+1)}\left(J_{2}, J_{3}\right) .
\end{aligned}
$$

Hence, the sums of components are

$$
\begin{aligned}
\Sigma_{2 k} & =b_{2 k} 2^{k+1}(\zeta+3)^{k} H_{2 k} H_{2(k+1)}\left(J_{2}, J_{3}\right), \\
\bar{\Sigma}_{2 k+1} & =b_{2 k+1} 2^{k+1}(\zeta+3)^{k+1} H_{2(k+1)}\left(J_{2}\right) H_{2(k+1)}\left(J_{3}\right) .
\end{aligned}
$$

To find the signs $b_{2 k}$ and $b_{2 k+1}$, we consider the limit $\zeta \rightarrow \infty$. On the one hand, Lemma 3.20 implies

$$
\begin{aligned}
H_{2 k} & =\zeta^{k(k-1)}(1+o(1)), & H_{2 k}\left(J_{2}\right) & =2^{1-k} \zeta^{k(k-1)}(1+o(1)), \\
H_{2 k}\left(J_{3}\right) & =\zeta^{k(k-1)}(1+o(1)), & H_{2 k}\left(J_{2}, J_{3}\right) & =2^{1-k} \zeta^{k(k-1)}(1+o(1)),
\end{aligned}
$$

and therefore

$$
\Sigma_{2 k}=2 b_{2 k} \zeta^{k(2 k+1)}(1+o(1)), \quad \bar{\Sigma}_{2 k+1}=2 b_{2 k+1} \zeta^{(k+1)(2 k+1)}(1+o(1)) .
$$


On the other hand, it follows from Lemma 4.11 that

$$
\Sigma_{2 k}=2 \zeta^{k(2 k+1)}(1+o(1)), \quad \bar{\Sigma}_{2 k+1}=2 \zeta^{(k+1)(2 k+1)}(1+o(1)) .
$$

We compare the coefficients of the leading terms and conclude that $b_{2 k}=b_{2 k+1}=1$.

Proposition 4.13 implies that the sums of components are polynomials in the parameter $\zeta$ with integer coefficients. As for the components studied in Sections 4.1 and 4.2, we compute the coefficients of its lowest- and highest-order term. For the lowest-order term, we recall that the number of diagonally- and antidiagonally-symmetric alternating sign matrices of size $2 n+1$ is given by [35]

$$
A_{\mathrm{DAD}}(2 n+1)=\prod_{i=0}^{n} \frac{(3 i) !}{(n+i) !} .
$$

Proposition 4.14. For each $k \geqslant 0$, we have

$$
\begin{aligned}
\Sigma_{2 k} & =2 A_{\mathrm{DAD}}(4 k+1)+\cdots+2 \zeta^{k(2 k+1)}, \\
\bar{\Sigma}_{2 k+1} & =2 A_{\mathrm{DAD}}(4 k+3)+\cdots+2 \zeta^{(k+1)(2 k+1)} .
\end{aligned}
$$

Proof. Both the coefficient and the exponent of the highest-order term follow from (4.103). To find the coefficient of the lowest-order term, we compute the sums of components for $\zeta=0$ :

$$
\begin{aligned}
\left.\Sigma_{2 k}\right|_{\zeta=0} & =\left.\left.2^{k+1} 3^{k} H_{2 k}\right|_{\zeta=0} H_{2(k+1)}\left(J_{2}, J_{3}\right)\right|_{\zeta=0}, \\
\left.\bar{\Sigma}_{2 k+1}\right|_{\zeta=0} & =\left.\left.2^{k+1} 3^{k+1} H_{2(k+1)}\left(J_{2}\right)\right|_{\zeta=0} H_{2(k+1)}\left(J_{3}\right)\right|_{\zeta=0} .
\end{aligned}
$$

To evaluate these expressions, we need (4.22) and (4.65), as well as [15]

$$
\left.H_{2 k}\left(J_{2}, J_{3}\right)\right|_{\zeta=0}=2^{-k} A_{\mathrm{UU}}^{(2)}(4 n ; 1,1,1),
$$

where the numbers

$$
A_{\mathrm{UU}}^{(2)}(4 n ; 1,1,1)=2^{2 n} \prod_{i=1}^{n} \frac{(6 i-1)(6 i-3) !}{(2(n+i)) !}
$$

appear in the enumeration of alternating sign matrices with two $U$-turn boundaries [29]. The final expression for the lowest-order coefficient is a result of the combinatorial identities

$$
\begin{aligned}
& A_{\mathrm{DAD}}(4 k+1)=3^{k} A_{\mathrm{V}}(2 k+1) A_{\mathrm{UU}}^{(2)}(4 k ; 1,1,1), \\
& A_{\mathrm{DAD}}(4 k+3)=3^{k+1} N_{8}(2(k+1)) A(2 k+1) / A_{\mathrm{V}}(2 k+1) .
\end{aligned}
$$

They follow, for example, from a factorisation of Schur functions into symplectic and orthogonal characters discussed in [35].

We note that the lowest-order term also follows from a rigorous result on the six-vertex model [36].

\subsection{Discussion}

In this section, we consider the eigenvalue problem

$$
H|\phi\rangle=E_{0}|\phi\rangle, \quad P|\phi\rangle=|\phi\rangle, \quad|\phi\rangle \in V^{2 n+1},
$$


for the XYZ Hamiltonian (4.77). Its space of solutions is one-dimensional [3]. We work with the solution

$$
\left|\phi_{n}\right\rangle=\frac{1}{2}\left(\left|\psi_{n}\right\rangle+\left|\bar{\psi}_{n}\right\rangle\right)
$$

Our goal is to compare our results for $\left|\phi_{n}\right\rangle$ to several conjectures by Bazhanov and Mangazeev [13], and Razumov and Stroganov [14], on their solutions, which we denote by $\left|\phi_{n}^{\mathrm{BM}}\right\rangle$ and $\left|\phi_{n}^{\mathrm{RS}}\right\rangle$, respectively. We refer to these conjectures as BM-Conjectures and RS-Conjectures. The comparison to our results suggests that $\left|\phi_{n}^{\mathrm{BM}}\right\rangle=\left|\phi_{n}^{\mathrm{RS}}\right\rangle=\left|\phi_{n}\right\rangle$, but this conclusion remains non-rigorous (even if we admit Conjecture 2.1). The main reason is that the normalisation conventions for $\left|\phi_{n}^{\mathrm{BM}}\right\rangle$ and $\left|\phi_{n}^{\mathrm{RS}}\right\rangle$ differ from the one for $\left|\phi_{n}\right\rangle$. We recall the normalisation of $\left|\phi_{n}\right\rangle$ is implicitly fixed by (2.29) and (2.30), and by the homogeneous limit (4.1) and (4.2). In the case of Bazhanov and Mangazeev's work, another reason is that the exact link between the families of polynomials they use and ours is still lacking.

\section{The BM-Conjectures}

The vector $\left|\phi_{n}^{\mathrm{BM}}\right\rangle$ is normalised so that its components are polynomials in $\zeta$ without a common polynomial factor. This fixes the vector up to an overall numerical factor. Its value is set by the additional requirement

$$
\left.\left(\phi_{2 k}^{\mathrm{BM}}\right)_{\underbrace{\uparrow \cdots \uparrow}_{2 k} \underbrace{\downarrow \cdots \downarrow}_{2 k+1}}^{{ }^{1}}\right|_{\zeta=0}=1,\left.\quad\left(\phi_{2 k+1}^{\mathrm{BM}}\right) \underbrace{\uparrow \cdots \uparrow}_{2 k+2} \underbrace{\downarrow \cdots \downarrow}_{2 k+1}\right|_{\zeta=0}=1 .
$$

Bazhanov and Mangazeev formulate their conjectures on $\left|\phi_{n}^{\mathrm{BM}}\right\rangle$ in terms of two families of polynomials $s_{n}(z), \bar{s}_{n}(z)$, where $n \in \mathbb{Z}$, with integer coefficients. These polynomials are solutions to a difference-differential equation [13]. The polynomials $s_{n}(z)$ are conjectured to possess the factorisation properties

$$
\begin{aligned}
s_{2 k+1}\left(y^{2}\right) & =\bar{c}_{2 k+1} p_{k}(y) p_{k}(-y), \\
s_{2 k}\left(y^{2}\right) & =\bar{c}_{2 k} \bar{p}_{k+1}(y) q_{k-1}(y),
\end{aligned}
$$

for each $k \in \mathbb{Z}$. Here, $p_{k}(y), q_{k}(y)$ are polynomials with integer coefficients, and $\bar{c}_{n}$ are known constants. Moreover, we use the abbreviation

$$
\bar{p}_{k}(y)=\left(\frac{1+3 y}{2}\right)^{k(k-1)} p_{-k}\left(\frac{y-1}{1+3 y}\right) .
$$

In his investigations of BM-Conjecture 1, Zinn-Justin [15] observed for small $k$ the relations

$$
H_{2 k}=\zeta^{k(k-1)} q_{k-1}\left(\zeta^{-1}\right), \quad 2^{k-1} H_{2 k}\left(J_{2}, J_{3}, J_{4}\right)=\zeta^{k(k-1)} q_{-k}\left(\zeta^{-1}\right)
$$

and

$$
\begin{aligned}
H_{2 k}\left(J_{2}\right) & =\zeta^{k(k-1)} \bar{p}_{-k+1}\left(\zeta^{-1}\right), & H_{2 k}\left(J_{3 / 4}\right)=\zeta^{k(k-1)} p_{k-1}\left( \pm \zeta^{-1}\right), \\
2^{k-1} H_{2 k}\left(J_{2}, J_{3 / 4}\right) & =\zeta^{k(k-1)} p_{-k}\left(\mp \zeta^{-1}\right), & 2^{k-1} H_{2 k}\left(J_{3}, J_{4}\right)=\zeta^{k(k-1)} \bar{p}_{k}\left(\zeta^{-1}\right) .
\end{aligned}
$$

We assume that they hold for arbitrary $k$, and use them to discuss BM-Conjecture 2, 3 and 4 .

BM-Conjecture 3 provides the explicit expression

$$
\left(\phi_{n}^{\mathrm{BM}}\right)_{\downarrow \cdots \downarrow}=\zeta^{n(n+1) / 2} s_{n}\left(\zeta^{-2}\right)
$$


for the component labelled by a polarised spin configuration. Using Proposition 4.7, we obtain the same component for the vector $\left|\phi_{n}\right\rangle$. For each $k \geqslant 0$, we have

$$
\left(\phi_{2 k}\right)_{\downarrow \cdots \downarrow}=\zeta^{k} H_{2 k} H_{2(k+1)}\left(J_{3}, J_{4}\right), \quad\left(\phi_{2 k+1}\right)_{\downarrow \cdots \downarrow}=\zeta^{k+1} H_{2(k+1)}\left(J_{3}\right) H_{2(k+1)}\left(J_{4}\right) .
$$

From the factorisation properties (4.116), the relations $(4.118)$, and $\bar{c}_{2 k+1}=1, \bar{c}_{2 k}=2^{-k}$ for $k \geqslant 0$ [13], we conclude that

$$
\left(\phi_{n}^{\mathrm{BM}}\right)_{\downarrow \cdots \downarrow}=\left(\phi_{n}\right)_{\downarrow \cdots \downarrow} .
$$

Likewise, BM-Conjecture 4 gives an explicit expression for the component labelled by an alternating spin configuration: For each $k \geqslant 0$,

$$
\begin{aligned}
\left(\phi_{2 k}^{\mathrm{BM}}\right)_{\uparrow \downarrow \cdots \uparrow \downarrow \downarrow} & =2^{k} \zeta^{2 k(k-1)} \bar{p}_{-(k-1)}\left(\zeta^{-1}\right) q_{k-1}\left(\zeta^{-1}\right), \\
\left(\phi_{2 k+1}^{\mathrm{BM}}\right)_{\uparrow \uparrow \downarrow \cdots \uparrow \downarrow} & =2^{k} \zeta^{2 k^{2}} \bar{p}_{-k}\left(\zeta^{-1}\right) q_{k-1}\left(\zeta^{-1}\right) .
\end{aligned}
$$

Similarly, we infer from Proposition 4.2 (and from the invariance of $\left|\phi_{n}\right\rangle$ under translations [3]) the components

$$
\left(\phi_{2 k}\right) \uparrow \downarrow \cdots \uparrow \downarrow \downarrow=2^{k} H_{2 k} H_{2 k}\left(J_{2}\right), \quad\left(\phi_{2 k+1}\right) \uparrow \uparrow \downarrow \cdots \uparrow \downarrow=2{ }^{k} H_{2 k} H_{2(k+1)}\left(J_{2}\right) .
$$

We use (4.118) to compare these expressions to (4.122), and find

$$
\left(\phi_{2 k}^{\mathrm{BM}}\right)_{\uparrow \downarrow \cdots \uparrow \downarrow \downarrow}=\left(\phi_{2 k}\right)_{\uparrow \downarrow \cdots \uparrow \downarrow \downarrow}, \quad\left(\phi_{2 k+1}^{\mathrm{BM}}\right)_{\uparrow \uparrow \downarrow \cdots \uparrow \downarrow}=\left(\phi_{2 k+1}\right)_{\uparrow \uparrow \downarrow \cdots \uparrow \downarrow}
$$

Since the space of solutions to the eigenvalue problem (4.113) is one-dimensional, the equalities (4.121) and (4.124) suggest that $\left|\phi_{n}^{\mathrm{BM}}\right\rangle=\left|\phi_{n}\right\rangle$. A proof of this equality would imply that all the components of $\left|\phi_{n}\right\rangle$ are polynomials in $\zeta$. This proof is, however, beyond the scope of this article. Nonetheless, it is interesting to explore the consequences of this equality. In BM-Conjecture 2, Bazhanov and Mangazeev claim that

$$
\left(\phi_{n}^{\mathrm{BM}}\right)_{\uparrow \cdots \uparrow \downarrow}=\frac{1}{2 n+1} \zeta^{n(n-1) / 2} \bar{s}_{n}\left(\zeta^{-2}\right) .
$$

We obtain the corresponding component of $\left|\phi_{n}\right\rangle$ from Proposition 4.7. For $k \geqslant 0$, we find

$$
\begin{aligned}
\left(\phi_{2 k}\right) \uparrow \cdots \uparrow \downarrow & =\frac{1}{2} \zeta^{k}\left((1+\zeta) H_{2 k}\left(J_{3}\right) H_{2(k+1)}\left(J_{4}\right)+(1-\zeta) H_{2 k}\left(J_{4}\right) H_{2(k+1)}\left(J_{3}\right)\right), \\
\left(\phi_{2 k+1}\right)_{\uparrow \cdots \uparrow \downarrow} & =\frac{1}{2} \zeta^{k}\left(\left(1-\zeta^{2}\right) H_{2(k+1)} H_{2(k+1)}\left(J_{3}, J_{4}\right)+H_{2 k} H_{2(k+2)}\left(J_{3}, J_{4}\right)\right),
\end{aligned}
$$

respectively. We match these expressions to (4.125) and find, for $k \geqslant 0$,

$$
\begin{aligned}
\bar{s}_{2 k}\left(y^{2}\right) & =\frac{(4 k+1)}{2 y^{2 k+1}}\left((1+y) p_{k-1}(y) p_{k}(-y)-(1-y) p_{k-1}(-y) p_{k}(y)\right), \\
\bar{s}_{2 k+1}\left(y^{2}\right) & =\frac{(4 k+3)}{\left(2 y^{2}\right)^{k+1}}\left(\left(y^{2}-1\right) q_{k}(y) \bar{p}_{k+1}(y)+\frac{1}{2} q_{k-1}(y) \bar{p}_{k+2}(y)\right) .
\end{aligned}
$$

These relations resemble the factorisations (4.116) for $s_{n}\left(y^{2}\right)$. To our best knowledge, they have not been reported in the literature. 


\section{The RS-Conjectures}

The vector $\left|\phi_{n}^{\mathrm{RS}}\right\rangle$ is normalised so that its components are polynomials in $\zeta$ without a common polynomial factor, too. ${ }^{4}$ Razumov and Stroganov argue that this normalisation convention implies that the component of highest degree is $\left(\phi_{n}^{\mathrm{RS}}\right)_{\downarrow \cdots \downarrow}$. According to RS-Conjecture 4.2, its highest-order term is

$$
\left(\phi_{n}^{\mathrm{RS}}\right)_{\downarrow \cdots \downarrow}=a_{n} \zeta^{n(n+1) / 2}+\cdots,
$$

where $a_{n}$ is non-zero and $\cdots$ denotes lower-order terms. Razumov and Stroganov consider $a_{n}=1$, which fixes the remaining overall numerical factor in their normalisation. By RS-Conjecture 4.4, this choice implies

$$
\begin{aligned}
\left(\phi_{2 k}^{\mathrm{RS}}\right)_{\downarrow \cdots \downarrow} & =A_{\mathrm{V}}(2 k+1)^{2} \zeta^{k}+\cdots+\zeta^{k(2 k+1)}, \\
\left(\phi_{2 k+1}^{\mathrm{RS}}\right)_{\downarrow \cdots \downarrow} & =N_{8}(2 k+2)^{2} \zeta^{k+1}+\cdots+\zeta^{(k+1)(2 k+1)} .
\end{aligned}
$$

Using Proposition 4.8, we find exactly the same expressions for the components $\left(\phi_{2 k}\right)_{\downarrow \cdots \downarrow}$ and $\left(\phi_{2 k+1}\right)_{\downarrow \cdots \downarrow}$, which suggests (but does not prove) the equality $\left|\phi_{n}^{\mathrm{RS}}\right\rangle=\left|\phi_{n}\right\rangle$. Another property supporting this equality is the sum rule

$$
\sum_{\boldsymbol{\alpha}}\left(\phi_{n}^{\mathrm{RS}}(\zeta)\right)_{\boldsymbol{\alpha}}=2^{-n(n-1) / 2}(\zeta-1)^{n(n+1) / 2}\left(\phi_{n}^{\mathrm{RS}}\left(\zeta^{\prime}\right)\right)_{\downarrow \cdots \downarrow}
$$

where $\zeta^{\prime}=(\zeta+3) /(\zeta-1)$, which is formulated in RS-Conjecture 5.2. It, indeed, holds for $\left|\phi_{n}\right\rangle$ as well, which straightforwardly follows from Proposition 4.13 and its proof.

\section{Conclusion}

In this article, we have investigated several scalar products involving a particular eigenvector of the transfer matrix of the inhomogeneous supersymmetric eight-vertex model with periodic boundary conditions. We have found explicit expressions for them in terms of the elliptic Tsuchiya determinant. In the homogeneous limit, they allowed us to compute the scalar products $S_{n}$ and $\bar{S}_{n}^{ \pm}$involving the basis vectors $\left|\psi_{n}\right\rangle,\left|\bar{\psi}_{n}\right\rangle$ of the eigenspace for the transfer-matrix eigenvalue $\Theta_{n}$ of the homogeneous eight-vertex model. Our main results are Theorems 4.1 and 4.6. They provide new and explicit expressions for these scalar products in terms of special polynomials introduced by Zinn-Justin and Rosengren. We have used them to compute several components of the basis vectors, as well as the sum of their components, establish their polynomial nature and compute their trigonometric limit.

We now discuss several open problems and generalisations of the present work. First, our results assume that Conjecture 2.1 holds. The proof of this conjecture remains a challenge, which would undoubtedly provide even more insight into the properties of the eigenvector $\left|\Psi_{n}\right\rangle$ and its homogeneous limit. Second, the comparison of our results to the investigations of Bazhanov and Mangazeev [13], and Razumov and Stroganov [14], strongly suggests that all the components of $\left|\psi_{n}\right\rangle$ are polynomials in $\zeta$ with integer coefficients. Proving the polynomial nature in $\zeta$ would be a step forward to settling most (if not all) of their conjectures. Third, we observe for small $n$ that the integer coefficients of the powers of $\zeta$ in a given component of $\left|\psi_{n}\right\rangle$ all have the same sign. This observation suggests that they could have a combinatorial meaning. We note that Hietala has recently found a combinatorial interpretation of the polynomial $H_{2 k}$ in terms of a partition function of the three-colour model [37]. Similar results for $H_{2 k}\left(J_{i}\right), H_{2 k}\left(J_{i}, J_{j}\right), \ldots$ remain to

\footnotetext{
${ }^{4}$ Razumov and Stroganov do not explicitly mention the absence of a common polynomial factor, but this assumption appears to be implicit in their work.
} 
be found. Fourth, the results of this article suggest that an exact finite-size computation of the emptiness or boundary emptiness formation probability for the supersymmetric eight-vertex model could be possible. In the trigonometric limit, these correlation functions are known $[36,38]$. Finally, we mention that there is a conjecture for a simple eigenvalue of the transfer matrix of the inhomogeneous supersymmetric eight-vertex model with open boundary conditions [39]. A characterisation of its eigenspace, similar to Conjecture 2.1, is still to be found.

\section{Acknowledgements}

This work was supported by the Fonds de la Recherche Scientifique-FNRS and the Fonds Wetenschappelijk Onderzoek-Vlaanderen (FWO) through the Belgian Excellence of Science (EOS) project no. 30889451 "PRIMA - Partners in Research on Integrable Models and Applications". SB is supported by the FNRS aspirant fellowship FC33665. We thank Jules Lamers, Jean Liénardy and Hjalmar Rosengren for discussions. Furthermore, $\mathrm{CH}$ thanks the Laboratoire de Physique Théorique des Modèles Statistiques, Orsay, France, where part of this work was done, for hospitality.

\section{References}

[1] Y. Stroganov, The importance of being odd, J. Phys. A: Math. Gen. 34 (2001) L179-L185.

[2] C. Hagendorf and P. Fendley, The eight-vertex model and lattice supersymmetry, J. Stat. Phys. 146 (2012) 1122-1155.

[3] C. Hagendorf and J. Liénardy, On the transfer matrix of the supersymmetric eight-vertex model. I. Periodic boundary conditions, J. Stat. Mech. (2018) 033106.

[4] R.J. Baxter, Solving models in statistical mechanics, Adv. Stud. Pure Math. 19 (1989) 95-116.

[5] K. Fabricius and B. M. McCoy, New developments in the eight vertex model II. Chains of odd length, J. Stat. Phys. 120 (2005) 37-70.

[6] H. Rosengren, Elliptic pfaffians and solvable lattice models, J. Stat. Mech. (2016) P083106.

[7] V.V. Bazhanov and V.V. Mangazeev, Eight-vertex model and non-stationary Lamé equation, J. Phys. A: Math. Gen. 38 (2005) L145-L153.

[8] V.V. Bazhanov and V.V. Mangazeev, The eight-vertex model and Painlevé VI, J. Phys. A: Math. Gen. 39 (2006) 12235-12243.

[9] H. Rosengren, Special polynomials related to the supersymmetric eight-vertex model. I. Behaviour at cusps, arXiv:1305.0666 (2013).

[10] H. Rosengren, Special polynomials related to the supersymmetric eight-vertex model. II. Schrödinger equation, arXiv:1312.5879 (2013).

[11] H. Rosengren, Special polynomials related to the supersymmetric eight-vertex model. III. Painlevé VI equation, arXiv:1405.5318 (2014).

[12] H. Rosengren, Special polynomials related to the supersymmetric eight-vertex model: A summary, Comm. Math. Phys. 340 (2015) 1143-1170. 
[13] V.V. Mangazeev and V.V. Bazhanov, The eight-vertex model and Painlevé VI equation II: eigenvector results, J. Phys. A: Math. Theor. 43 (2010) 085206.

[14] A.V. Razumov and Yu.G. Stroganov, A possible combinatorial point for the XYZ spin chain, Theor. Math. Phys. 164 (2010) 977-991.

[15] P. Zinn-Justin, Sum rule for the eight-vertex model on its combinatorial line, in K. Iohara, S. Morier-Genoud and B. Rémy (eds.), Symmetries, Integrable Systems and Representations, Volume 40, 599-637, Springer London (2013).

[16] V.E. Korepin, N.M. Boboliubov and A.G. Izergin, Quantum inverse scattering method and correlation functions, Cambridge University Press (1993).

[17] A.G. Izergin, D.A. Coker and V.E. Korepin, Determinant formula for the six-vertex model, J. Phys. A: Math. Gen. 25 (1992) 4315-4334.

[18] R.J. Baxter, Exactly solved models in statistical mechanics, London Academic (1982).

[19] E.T. Whittaker and G. N. Watson, A course of modern analysis, Cambridge University Press (1927).

[20] I.S. Gradshteyn and I.M. Ryzhik, Table of integrals, series and products, Elsevier (2007).

[21] T. Inami and H. Konno, Integrable XYZ spin chain with boundaries, J. Phys. A : Math. Gen. 27 (1994) L913-L918.

[22] H. Rosengren, An Izergin-Korepin-type identity for the 8VSOS model, with applications to alternating sign matrices, Adv. Appl. Math. 43 (2009) 137-155.

[23] L. Ahlfors, Complex analysis, McGraw-Hill (1979).

[24] G. Filali, Elliptic dynamical reflection algebra and partition function of SOS model with reflecting end, J. Geom. Phys 61 (2011) 1789 - 1796.

[25] D. Bressoud, Proofs and confirmations: The story of the alternating sign matrix conjecture, Cambridge University Press (1999).

[26] H. Weber, Lehrbuch der Algebra, Band 3: Elliptische Funktionen und algebraische Zahlen, Vieweg und Sohn (1908).

[27] M. Noumi, Painlevé equations through symmetry, American Mathematical Society (2004).

[28] R.E. Behrend, P. Di Francesco and P. Zinn-Justin, On the weighted enumeration of alternating sign matrices and descending plane partitions, J. Comb. Theor. 119 (2012) 331 - 363.

[29] G. Kuperberg, Symmetry classes of alternating-sign matrices under one roof, Ann. Math. 156 (2002) 835-866.

[30] A.V. Razumov and Yu.G. Stroganov, Refined enumerations of some symmetry classes of alternating-sign matrices, Theor. Math. Phys. 141 (2004) 1609-1630.

[31] A.V. Razumov, Yu.G. Stroganov and P. Zinn-Justin, Polynomial solutions of qKZ equation and ground state of $X X Z$ spin chain at $\Delta=-1 / 2$, J. Phys. A: Math. Gen. 40 (2007) 11827.

[32] A. Ayyer and R.E. Behrend, Factorization theorems for classical group characters, with applications to alternating sign matrices and plane partitions, J. Comb. Theor. A 165 (2019) $78-105$. 
[33] S. Okada, Enumeration of symmetry classes of alternating sign matrices and characters of classical groups, J. Alg. Comb. 23 (2006) 43-69.

[34] S. Brasseur, The eight-vertex model on its combinatorial line, Master's thesis, Université catholique de Louvain (2019).

[35] R.E. Behrend, I. Fischer and M. Konvalinka, Diagonally and antidiagonally symmetric alternating sign matrices of odd order, Adv. Math. 315 (2017) $324-365$.

[36] A. Morin-Duchesne, C. Hagendorf and L. Cantini, Boundary emptiness formation probabilities in the six-vertex model at $\Delta=-1 / 2$, J. Phys. A: Math. Theor. 53 (2020) 255202.

[37] L. Hietala, A combinatorial description of certain polynomials related to the XYZ spin chain, SIGMA 16 (2020) 101.

[38] L. Cantini, Finite size emptiness formation probability of the $X X Z$ spin chain at $\Delta=-1 / 2$, J. Phys. A: Math. Theor. 45 (2012) 135207.

[39] C. Hagendorf and J. Liénardy, On the transfer matrix of the supersymmetric eight-vertex model. II. Open boundary conditions, J. Stat. Mech. (2020) 033104. 